\title{
Strain Tensor Determination of Compressed Individual Silica Sand Particles Using High-Energy Synchrotron Diffraction
}

\author{
Khalid Alshibli ${ }^{1}$, Mehmet B. Cil $^{2}$, Peter Kenesei ${ }^{3}$, Ulrich Lienert ${ }^{4}$
}

Corresponding Author:

Dr. Khalid Alshibli, Dept. of Civil \& Env. Engineering, 73A Perkins Hall, University of Tennessee, Knoxville, TN 37996, USA, Tel. 001-865-974-7728, Fax: 001-865-974-2669, Email: Alshibli@utk.edu

\section{Published at:}

Alshibli, K. A., Cil, M. B., Kenesei, P., and Lienert, U. (2013) "Strain Tensor Determination of Compressed Individual Silica Sand Particles Using High-Energy Synchrotron Diffraction", Granular Matter, Vol. 15, pp. 517-530, DOI: 10.1007/s10035-013-0424-X

Keywords: Silica Sand, Lattice strain, 3D x-ray diffraction, granular materials, synchrotron

\footnotetext{
${ }^{1}$ Professor, Dept. of Civil \& Env. Engineering, University of Tennessee, Knoxville, TN 37996, Email: Alshibli@utk.edu

${ }^{2}$ Graduate student, Dept. of Civil \& Env. Engineering University of Tennessee, Knoxville, TN 37996, Email: mcil@utk.edu

${ }_{3}^{3}$ Research Scientist, Argonne National Laboratory, Argonne, IL, Email: kenesei@aps.anl.gov

${ }^{4}$ Research Scientist, Deutsches Elektronen-Synchrotron, DESY Photon Science, Hamburg, Germany, Email: ulrich.lienert@desy.de
} 


\begin{abstract}
The three-dimensional $\mathrm{x}$-ray diffraction (3DXRD) nondestructive technique was used to measure lattice strains within individual sand particles subjected to compressive loading. Three experiments were conducted on similar single columns of silica sand particles with particle sizes between $0.595 \mathrm{~mm}$ and $0.841 \mathrm{~mm}$. In each experiment, three sand particles were placed inside an acrylic mold with an inner diameter of $1 \mathrm{~mm}$. Multiple in situ 3DXRD scans were acquired for each sand column as compressive load was increased. The volume-averaged lattice strain tensor was calculated for each sand particle. In addition, particle orientation and volumetric strain were calculated for individual sand particles. The axial normal strain $\varepsilon_{\mathrm{zz}}$ exhibited a linear response in the range of 0 to $10^{-3}$ when the applied compressive axial load (F) increased from 0 to $\sim 30 \mathrm{~N}$ when one particle in the sand column fractured. Stress tensor of individual particles was calculated from the acquired lattice strain measurements and elastic constants of silica sand that were reported in the literature. To the best of our knowledge, there have been no reported experimental measurements of the lattice strain tensor measurements within individual silica sand particles. The quantitative measurements reported in this paper at the particle level are very valuable for developing, validating or calibrating micromechanics-based finite element and discrete element models to predict the constitutive behavior of granular materials. 3DXRD represents an exciting new non-destructive technique to directly measure constitutive behavior at the scale of individual particles.
\end{abstract}




\section{INTRODUCTION}

The constitutive behavior and deformation characteristics of uncemented granular materials are to a large extent derived from the fabric or geometry of the particle structure and inter-particle friction resulting from normal forces acting on particles or particle groups. Granular materials consist of discrete particles with fabric (microstructure) that changes under loading. Microstructure (or fabric) is defined as the arrangement of particles, particle groups, and the associated pore space. Extensive research has been conducted to describe the constitutive behavior and deformation characteristics of granular materials using the theory of plasticity [1,2], micro-polar theory [3-5], discrete element method (DEM) [6-8], and coupled continuum-DEM models [9]. Some of these models provide excellent predictions of the strength behavior and deformation mode of granular materials; however, despite recent technological advances, calibration of such models using representative material parameters has always been a challenge.

In the last few decades, several experimental techniques were utilized to capture the particle-to-particle interaction under shear/uniaxial loading. Most of these efforts were limited to shearing relatively large cylindrical rods or circular/oval discs under simple shear or plane stress/strain loading configurations and used optical methods to track deformations [10-12]. Some investigations involved stabilizing the specimen with epoxy, cutting thin sections, and using two-dimensional (2D) microscopy to study the internal fabric of granular materials. Photoelastic materials were also used to visualize stress distribution and force chains in granular materials [13-16]; however, it is very difficult to extract force values from these images. Furthermore, all research using photoelasticity was applied to 2D loading conditions.

X-ray computed tomography (CT) has emerged as a powerful non-destructive 3D scanning technique to study geomaterials. Alshibli and Reed [17] compiled papers that report the latest 
advances in research on applying CT to characterize properties of geomaterials. Synchrotron micro-computed tomography (SMT) offers a significant enhancement to 3D imaging when compared to industrial CT systems by producing images with a higher resolution (in the order of 5 micron/voxel). For example, Hasan and Alshibli [18] acquired excellent measurements of particle translation and rotation and the change of fabric during shearing of silica sand. Although CT and SMT can produce a 3D density map of the scanned specimen that can be used to identify particle position, morphology, and void space, they cannot provide measurements of contact stresses and strain measurement at particle contacts.

Neutron diffraction tomography, which can also yield 3D images of scanned objects, is more sensitive to hydrogen content and has better penetration power through metal containers surrounding the specimen. It is ideal for applications that involve detection of water within the scanned specimen. Neutrons are seldom absorbed by the sample as assumed in the radon transformation, but are more often scattered by elastic and inelastic collisions with nuclei and phonons [19]. Still, the transformation yields very useful 3D images. Most of the neutron diffraction images in the literature are blurred with a resolution an order of magnitude lower than CT images. For example, Penumadu et al. [20] used neutron diffraction to measure lattice strains in the bulk of silica sand and found a significant difference between the global and lattice strainstress relationship. Frischbutter et al. [21] used the same technique to measure lattice strain in geological samples and found a significant difference between lattice strain and strain measured by strain gauges. Darling et al. [22] reported similar measurements and reached similar conclusions.

Three-dimensional $\mathrm{x}$-ray diffraction (3DXRD) microscopy is a promising technique that has the potential to measure lattice strains and crystallographic orientation of crystalline 
materials. It is based on transmission geometry that can be applied in both layered and bulk materials [23-27]. It allows nondestructive 3D mapping of polycrystalline materials and monitors in situ dynamic processes of the bulk specimens. It also permits structural characterization of individual grains embedded in polycrystals. The volume, strain, phase, and orientation of hundreds of grains/particles can be monitored simultaneously [28]. 3DXRD represents a new opportunity to directly acquire information about the interaction of individual particles. Current applications of 3DXRD focus primarily on polycrystalline metals. Poulsen [25] introduced 3DXRD technique and its potential applications in materials science. Martins et al. [29] measured the elastic strains of individual aluminum grains as a function of tensile load. Jensen et al. [28] demonstrated that 3DXRD could be used to monitor recrystallization in metallurgical processes, grain migration during recrystallization, solid-state nucleation, and grain growth during phase transformations. Hall et al. [30] presented preliminary results of 3DXRD scanning on Ottawa sand specimens that were subjected to 1D loading. However, their analysis did not include extracting lattice strain tensor measurements from the data.

The main objective of this paper is to determine volume-averaged lattice strain tensor components of individual silica sand particles under axial loading condition using the 3DXRD microscopy. Beamline 1-ID of the Advanced Photon Source (APS), Argonne National Laboratory, USA, was used to acquire the scans for three sand specimens. Analyzing 3DXRD data is typically a challenging task. The authors have developed evaluation codes in MATLAB augmented by open source scientific programs called Fable [31] and DIGIgrain developed by Kenesei [32] to analyze the data to extract lattice strain tensor values. To the best of our knowledge, there have been no reported experimental measurements of the lattice strain tensor measurements within individual silica sand particles. 


\section{PRINCIPLE OF 3DXRD MICROSCOPY}

The incident monochromatic synchrotron x-ray beam is scattered on the orderly arranged atoms of a crystalline material. The diffracted x-ray beams obey Bragg's law:

$$
2 d_{h k l} \sin \theta_{h k l}=\lambda
$$

where $\lambda$ is the wavelength of the incident beam, $\theta_{h k l}$ is the angle of the incident beam to the lattice planes with distance of $d_{h k l}$. The subscript $h k l$ denotes the lattice planes $(h k l)$ with Miller indices of $h, k$, and $l$. Accordingly, the diffracted beams can be observed only in the directions that fulfill the Bragg condition (Eq. 1) and constructive interference can be obtained as defined by selection rules on Miller indices according to the crystal symmetries and atomic form factors. A simple visual interpretation of the diffraction geometry is shown in Figure 1, where the incident x-ray beams are reflected on the set of lattice planes $(h k l)$ like on a mirror, and the angle of the diffracted beam to the incident beam is $2 \theta_{h k l}$. Thus, the diffracted peak positions from a powder-like sample are located on the Debye-Scherrer rings. The knowledge of wavelength $\lambda$, and the measurement of the diffraction angle $2 \theta_{h k l}$ provide information about the lattice spacing $d_{h k l}$, which is in direct correspondence with the lattice strain projected to the normal of the given lattice planes. A family of parallel lattice planes with spacing $d_{h k l}$ is described by a reciprocal lattice vector, later referred to as g-vector. As the applied compressive stress increases, the crystal gets distorted, and the lattice spacings (g-vectors) change according to the constitutive response of the material. More detailed information about the 3DXRD geometry can be found in the literature [25]. 
In the high-energy 3DXRD measurements (typically 50-100 keV x-ray), since the diffraction angles are usually less than $15^{\circ}$, several diffraction rings can be measured simultaneously using a large area detector. An additional benefit of applying high-energy x-rays is the high penetration through the sample, which enables investigation of millimeter-sized samples. Rotating the polycrystalline sample around the loading axis permits the detection of diffraction peaks from several sets of lattice planes of each crystallite, which fulfills the Bragg condition and provides information about several projections of the lattice strain to determine the volume-averaged strain tensor of individual particles.

\section{EXPERIMENTS}

The sand used in the experiments is natural uniform silica sand known as ASTM 20-30 Ottawa sand with particle size between $0.595 \mathrm{~mm}$ (Sieve No. 30) and $0.841 \mathrm{~mm}$ (Sieve No. 20). It was mined from Ottawa, Illinois, USA. To investigate the crystallographic structure of the sand particles, laboratory-based powder diffraction x-ray analysis was performed on a sample of the sand. Diffraction patterns of the sample showed that $\alpha$-quartz $\left(\mathrm{SiO}_{2}\right.$, Space group P3221) is the only detectable mineral in the sample, confirming the chemical composition as $99.8 \% \mathrm{SiO}_{2}$ as provided by the manufacturer, U.S. Silica Company. Lattice parameters of crystalline materials are usually determined using conventional XRD. Lattices are typically expressed using three lattice constants known as $a, b$, and $c$, and three angles $(\alpha, \beta, \gamma)$ between the lattice vectors in the 3D lattice space. We used XRD to measure the lattice constants of the sand powder as follows: $a$ $=4.916 \AA, b=4.916 \AA, c=5.4054 \AA$ and the angles are $\alpha=90^{\circ}, \beta=90^{\circ}, \gamma=120^{\circ}$. These values are very close to lattice constants of $\alpha$-quartz that were reported by Levien et al. [33]. 
Crystallographic orientation spread within individual sand particles was also investigated using polarized light microscopy. A sample of the sand was illuminated with the polarized light and rotated, and the change in the transmitted light was observed to determine the crystallographic perfection of the sand particles. During the rotation, the intensity of transmitted light seems to be uniform throughout the surface in most of the sand particles and only a few exhibited anisotropic optical behavior. These observations demonstrated that most of the particles had a nearly single crystal particle structure, which is in agreement with the findings of Hall et al. [30].

A special test cell was fabricated to conduct the experiments. Three sand particles were placed inside a $1 \mathrm{~mm}$ cylindrical hole at the center of a $15 \mathrm{~mm}$ acrylic cylindrical mold (Figure 2). The load was measured at the top and the bottom end plates using two load cells. The bottom load cell was attached to the aluminum base plate of the cell. An acrylic spacer was used to hold the cylindrical mold that contained the sand particles. The load of particles on the bottom load plate was transmitted through tungsten carbide rod with a diameter of $0.8 \mathrm{~mm}$ and a height of $12.7 \mathrm{~mm}$. The acrylic mold was placed on the top of the bottom loading plate extension without any friction between them. Then, three particles were placed inside the mold using a special aluminum funnel with $1 \mathrm{~mm}$ diameter. To prevent possible plugging or jamming of particles inside the $1 \mathrm{~mm}$ hole, only three particles were used in the experiments. The height of the sand column ranged from $1.7 \mathrm{~mm}$ to $2.4 \mathrm{~mm}$. Finally, the top loading plate was set in place and the test cell was assembled. This experimental setup permits the loads to be measured at the top and bottom of the sand column to quantify the influence of friction between sand particles and mold walls. 
The test cell was mounted on the sample stage of beamline 1-ID at APS. The specimen was subjected to axial loading at a constant displacement rate using a computerized stepper motor. The sand particles were illuminated by a high-energy $\mathrm{x}$-ray beam with a size of $0.8 \mathrm{~mm} \times$ $1.0 \mathrm{~mm}$ and an energy of $80.725 \mathrm{keV}$ that corresponds to wavelength of $0.15358 \AA$. Diffraction data were detected by an area detector that was positioned at a horizontal distance of $1593 \mathrm{~mm}$ ( $L$ in Figure 1b) from the specimen. The beam was centered on each particle in the sand column, and a rotation scan was acquired for a beam height of $0.8 \mathrm{~mm}$. In the case of the middle particle, for example, the beam height partially included the two adjacent sand particles. The sand column was scanned before the axial load was applied; it was then loaded until the desired compressive load was reached and the scan was performed while the stepper motor position was held. Multiple 3DXRD scans were acquired at the load levels shown in Figure 3a. Compression forces, displacement, stress, and strain are taken to be positive throughout this paper according to the sign convention in the field of geotechnical engineering. Figure $3 \mathrm{~b}$ shows an example SMT image series of a similar specimen in a similar loading test. The SMT images were acquired using Sector 13 beamline at APS and have proved very helpful in the selection of the load steps for the diffraction measurements.

Three specimens were scanned using 3DXRD at multiple loads. Each specimen consisted of three sand particles, which were loaded at different displacement rates $(0.0125 \mathrm{~mm} / \mathrm{min}, 0.05$ $\mathrm{mm} / \mathrm{min}$, and $0.20 \mathrm{~mm} / \mathrm{min}$ ). 3DXRD data were acquired while the specimen was rotated at a constant angular velocity about an axis perpendicular to the beam direction. An intensity distribution image of the diffracted spots (called the $\omega$-slice) was recorded at each rotation interval of $\Delta \omega$. The raw diffraction data consist of 180 stacked images recorded while the 
specimen was rotated $\omega$ from $-90^{\circ}$ to $90^{\circ}$ with $\Delta \omega=1^{\circ}$ integration interval at each loading stage for each particle.

\section{DIFFRACTION DATA ANALYSIS}

The scanning dataset represents a map of the $3 \mathrm{D}$ reciprocal space distribution of the investigated particle. Figure 4 depicts an example image of the diffraction data. The discrete peaks appeared at the location predicted by the Bragg condition as expected, and no significant overlap of the measured spots was observed. Figure 4 clearly demonstrates the behavior of the spots under the effect of progressive loading. Reflections appear and disappear at specific $\eta, \omega$, and $2 \theta$ positions as extended spots according to the imperfections and defects of the crystals. The deformation leads to displacement of spots on the detector according to the particle rotation and lattice distortion. Therefore, reflections may move through $\{\eta, \omega, 2 \theta\}$ space during the deformation process. Moreover, small particle fragments formed as a result of particle fracture and resulted in an increase in the number of spots.

As an example, Figure 5 provides $\eta-\omega$ maps of the center layer of the sand column. The images represent the measured diffraction spots recorded in the first Debye-Scherrer ring with $2 \theta_{\{100\}}=2.07^{\circ}$ at selected load steps. Before particle fracture, a significant orientation change or particle rotation occurs, then the particle disintegrates into several small fragments. These images show that the deformation causes certain changes in the structure of sand particles.

The analysis of the diffraction data consists of three primary stages: (i) preprocessing and peak search; (ii) g-vector calculation and indexing; and (iii) orientation; and strain computation and error analysis. The preprocessing and peak search steps were performed using the DIGIgrain 
program. In the preprocessing stage, DIGIgrain applies dark current and background correction on the raw data. After data correction, it uses a sophisticated 3D searching algorithm for segmenting the extended, sometimes slightly overlapping diffraction spots and evaluates the peak positions (center of the spot profile) with high precision taking into account the spatial distortion of the detector. The peak search algorithm offers many options for identifying the peaks properly and can be efficiently customized for various datasets. During the peak search, the peaks can be filtered by size, position, intensity, and shape, and the slightly overlapping peaks can be separated based on the 3D intensity profile of the spots. The DIGIgrain algorithm successfully eliminated the spot overlapping and spatial distortion effects and provided the reduced dataset for further analysis. The $2 \mathrm{D}$ raw diffraction data was reduced into a simple text file for each scan that includes a compact list of peaks and their properties such as center position, integrated intensity, statistical descriptors, etc.; these files were used as input for the Fable program.

Armed with the knowledge of the best possible peak positions, one can calculate the gvectors using the ImageD11 application of Fable [34], which considers the detector calibration parameters such as the detector tilt, distance, and pixel size determined from powder diffraction measurements on a calibrant $\left(\mathrm{LaB}_{6}\right)$. Then, the GrainSpotter application of Fable [35] can be used to assign the measured g-vectors to individual particles. This process is called indexing. GrainSpotter identifies the particle orientations via searching in the orientation space based on the value of completeness, which is defined as the ratio of the number of the found and expected g-vectors belonging to a particle. In this paper, approximately $150 \mathrm{~g}$-vectors were assigned to each particle per scan. The results were summarized in an output file that includes the center of mass position and orientation (in form of the U-matrix) of each detected particle, the angle 
between the theoretical and the measured diffraction vector (called internal angle), and the list of the diffraction spots assigned to the particle.

Silica sand particles rotate and translate significantly in real 3D space due to their discrete nature. Due to significant rotations of the investigated particles, assigning measured spots to a specific particle was challenging. Therefore, a visual validation process was implemented by comparing the spots identified by GrainSpotter and those measured during the diffraction scan. Figure 6 shows an example image of the spot matching for load step 2 and ring $2 \theta=2.07^{\circ}$ with a Miller index of $\{010\}$. GrainSpotter successfully indexed all spots that appeared in the $\eta-\omega$ maps. This visualization was repeated for consecutive load steps at different Debye-Scherrer rings. This evaluation process confirmed accurate indexing of particles and determined the sequence of particle identifiers in each loading step, resulting from the independent indexing procedure, to follow the orientation and strain history of the individual particles over the load steps. This visual matching method increased the confidence in the accuracy of the particle identification procedure.

\section{RESULTS}

\section{Track particle orientation}

The rotations of particles were evaluated analytically. The misorientation angle of the particle with reference to an orientation before loading was calculated based on the indexing results for each load step. A particle could rotate such that only the rotation axis changes but not the misorientation angle with respect to the reference orientation. In order to detect this change, $\Delta$ orientation which compares the actual orientation relative to the previous one instead of a fixed reference was also determined. The particle with the minimum misorientation angle was 
identified as the candidate for the reference particle in continuous loading. The result was also confirmed by comparing the detected spots of selected particles in each load step. An example development of the misorientation angle of a particle relative to the initial condition as a reference orientation and the variation of $\Delta$ orientation are depicted in Figure $7 \mathrm{a}$, which confirms the assumed significant rotation of the particle as a rigid body.

The rotation path of the middle particle of Test 3 is also illustrated in Figure $7 \mathrm{~b}$ in Rodrigues space. Rodrigues vectors are an axis/angle representation of orientations, the direction of the vector is parallel to the rotation axis and the length is proportional to the rotation angle around the axis (e.g. Morawiec and Field [36]). Rodrigues representation is chosen since the crystal symmetry is easily represented by the fundamental zone consisting of a hexagonal prism for the trigonal symmetry of quartz. Also, distortions of the distances between orientations are rather small in Rodrigues representation. In the present case, the particle orientation is fully contained in one octant of the fundamental zone which is shown in Figure $7 \mathrm{~b}$ together with projections onto the xyz planes of the sample coordinate system. It is seen that the $\mathrm{z}$-component is small compared to the in-plane xy-components which means that the particle mainly rotated around axes that are perpendicular to the loading direction. Second, initially the rotation path was smooth around a fixed axis (mostly the length of the Rodrigues vector changes, not the direction). Note that the diffraction approach provides an orientation resolution of about $0.1^{\circ}$ which is far better than SMT imaging of particles. However, diffraction data are subjected to the ambiguity set by point group symmetry (trigonal). Large orientation changes, which are expected when particles fracture, can only be determined to the uncertainty of crystal symmetry operations. For example, referring to Figure $7 \mathrm{~b}$, the discontinuity occurring at the fracture (between points \#8 and \#9) may indicate that the rotation path actually extends outside of the 
fundamental zone. However, the correct symmetry element could be identified from simultaneous SMT data since the external shape of the particle is observed rather than the orientation of the crystal lattice.

As mentioned earlier granular particles translate and rotate when they are sheared. Particle rotation and the associated interlocking and moment contribute significantly to the shearing resistance of a bulk of granular materials. The results of this paper demonstrated that 3DXRD offers an excellent tool to accurately measure particle rotation with a high accuracy $\left(\right.$ about $0.1^{\circ}$ ), which provides valuable measurements to calibrate constitutive models to better capture the behavior of granular materials.

\section{Lattice strain tensor measurements}

The lattice-strain calculation using 3DXRD data is based on the evaluation of the $2 \theta$ shifts of the peak positions, which is achieved by measuring the relative changes of reference-vector length to an unloaded lattice condition. The strain tensor components represent the average deformation of all unit cells within the particle as a function of the applied load. For each $g$ vector (indexed by $i$, where $i=1 \ldots N$ ) of a particle, the projected strain in a specific direction described by unit directional vectors $\left(l_{i}, m_{i}, n_{i}\right)$ can be expressed as follows [37]:

$$
\varepsilon_{i}=\left(\begin{array}{lll}
l_{i} & m_{i} & n_{i}
\end{array}\right) \hat{\varepsilon}\left(\begin{array}{c}
l_{i} \\
m_{i} \\
n_{i}
\end{array}\right)=\left(\begin{array}{lll}
l_{i} & m_{i} & n_{i}
\end{array}\right)\left(\begin{array}{ccc}
\varepsilon_{\mathrm{xx}} & \varepsilon_{\mathrm{xy}} & \varepsilon_{\mathrm{xz}} \\
\varepsilon_{\mathrm{xy}} & \varepsilon_{\mathrm{yy}} & \varepsilon_{\mathrm{yz}} \\
\varepsilon_{\mathrm{xz}} & \varepsilon_{\mathrm{yz}} & \varepsilon_{\mathrm{zz}}
\end{array}\right)\left(\begin{array}{c}
l_{i} \\
m_{i} \\
n_{i}
\end{array}\right)
$$

where $\varepsilon_{i}$ is calculated using the length of the g-vectors in the unloaded $\left(g_{i}^{0}\right)$ and loaded $\left(g_{i}\right)$ cases as: 


$$
\varepsilon_{i}=\frac{g_{i}-g_{i}^{0}}{g_{i}^{0}}
$$

As mentioned earlier, the number of the experimentally evaluated projected strains $N$ for one particle is on the order of 100, while the number of unknown strain tensor components is only 6 . Therefore, we can build an over-determined equation system to determine the unknown strain tensors, which can be solved for $\hat{\varepsilon}$ using the least-squares approach. For simplicity, we change to a contracted 6-component vector notation for the symmetric tensors at this point, as:

$$
\hat{\varepsilon} \rightarrow \tilde{\varepsilon}=\left(\begin{array}{llllll}
\varepsilon_{\mathrm{xx}} & \varepsilon_{\mathrm{yy}} & \varepsilon_{\mathrm{zz}} & 2 \varepsilon_{\mathrm{xy}} & 2 \varepsilon_{\mathrm{xz}} & 2 \varepsilon_{\mathrm{yz}}
\end{array}\right)^{\mathrm{T}}
$$

The projected strains after simplifying the right side of Equation (2) are:

$$
\varepsilon_{i}=\varepsilon_{\mathrm{xx}} l_{i}^{2}+\varepsilon_{\mathrm{yy}} m_{i}^{2}+\varepsilon_{\mathrm{zz}} n_{i}^{2}+2 \varepsilon_{\mathrm{xy}} l_{i} m_{i}+2 \varepsilon_{\mathrm{xz}} l_{i} n_{i}+2 \varepsilon_{\mathrm{yz}} m_{i} n_{i}
$$

by which the linear equation system to be solved can be written in short form as:

$$
\left\{\varepsilon_{i}\right\}=[\mathrm{A}] \tilde{\varepsilon}
$$

where $\left\{\varepsilon_{\mathrm{i}}\right\}$ denotes the $\mathrm{N}$-element vector constructed from the projected strains and $[\mathrm{A}]$ is an $\mathrm{N} \times 6$ component matrix constructed as:

$$
[\mathrm{A}]=\left\{\begin{array}{llllll}
l_{i}^{2} & m_{i}^{2} & n_{i}^{2} & 2 l_{i} m_{i} & 2 l_{i} n_{i} & 2 m_{i} n_{i}
\end{array}\right\}
$$

where the components are calculated from lattice g-vectors as:

$$
\left(\begin{array}{lll}
l_{i} & m_{i} & n_{i}
\end{array}\right)^{T}=\frac{\mathbf{g}_{i}}{\left|\mathbf{g}_{i}\right|}
$$

Since the scans were acquired by rotating the specimen from $-90^{\circ}$ to $90^{\circ}$, the set of planes that satisfy the Bragg condition gave rise to diffraction spot on the detector twice- - belonging to both $(\mathrm{h}, \mathrm{k}, \mathrm{l})$ and $(-\mathrm{h},-\mathrm{k},-\mathrm{l})$ indices-during this rotation. These symmetrical spots are called 
Friedel pairs, an example of which can be identified clearly in the $\eta-\omega$ map shown in Figure 6. Symmetrical spots appear in the $\eta$ ranges $\left[-90^{\circ}, 270^{\circ}\right]$ and $\left[270^{\circ}, 90^{\circ}\right]$. The g-vectors of the Friedel pair spots are anti-parallel and originate from the same lattice planes [38]. Averaging Friedel pairs reduces errors in experimental lengths of g-vectors. In this paper, including Friedel pairs in the analysis resulted in negligible differences in the strain tensor calculations due to the large number of measured g-vectors; therefore, we decided not to include them in the analysis.

Strain calculations were performed using a MATLAB script developed by the authors. The ideal $g$-vectors before loading were determined as the reference condition based on the lattice constants obtained from the powder diffraction. The change in the diffraction vectors in each load increment was determined by normalizing measurements with respect to the initial, supposedly strain-free, condition. The error that stems from the particle position relative to the rotation center was also considered in the analysis, and offsets of the particle position were also included. The number of $g$-vectors used in strain tensor computation ranged between 125 and 156 after filtering out the mistakenly indexed g-vectors.

3DXRD data were collected for three similar uniaxial compression experiments and were used to calculate the lattice strain tensors of individual sand particles. The experimental configuration and parameters were kept the same in the three tests with exception of the loading rate. The results of the three experiments are similar to each other. For brevity, only the results of Test 3 are presented in detail; the results of Tests 1 and 2 are only summarized in Table 1. Figure 8 presents the load-versus-lattice-strain relationships of the three sand particles of Test 3 , which displays the lattice strain components that were determined based on the global coordinate system (i.e., experiment coordinate system shown in Figure 1b). 
The top and bottom load cells were used to record the compressive loads at both ends of the sand column and therefore to determine the load transmitted by the sand column. The difference between the two load measurements represents the friction force between the particles and the walls of the acrylic tube. The difference in loads was less than $0.5 \mathrm{~N}$ in the three tests. Referring to Figure $8, \varepsilon_{\mathrm{zz}}$ represents the normal strain along the loading direction. It increased in a nearly linear fashion as the compressive load increased until one of the sand particles fractured (middle particle in Test 3). Once a sand particle fractures, the small fragments of the particle will have a relatively large translation and rotation and will produce multiple diffraction spots causing unreliable strain measurements for the fractured particle. Furthermore, one particle fracture leads to instant load drop and rearrangement of other particles in the system. These two complex mechanisms caused the drastic shift of the strain measurements after middle particle fracture. $\varepsilon_{\mathrm{xx}}$ and $\varepsilon_{\mathrm{yy}}$ represent normal strains in the lateral directions, which should exhibit similar trends if the sand particles exhibit isotropic behavior, centered in the mold and spherical (i.e., each sand particle will have continuous contact with the mold wall). However, as depicted in Figure $3 b$, the non-spherical shape of the particles and the small space between the mold walls and the particles, allowed the particles to establish contact with one side of the mold, and the increase in axial load resulted in compression strain (positive values) in one of the lateral directions (e.g., $\varepsilon_{\mathrm{xx}}$ in Figure 8) and expansion strain (negative values) in the other lateral direction ( $\varepsilon_{\mathrm{yy}}$ in Figure 8). In some cases, $\varepsilon_{\mathrm{xx}}$ and $\varepsilon_{\mathrm{yy}}$ changed trend direction (i.e., switched from compression to tension or vice versa) as the test proceeded because some sand particles translated and rotated as the load increased, which changed the stress at the particle-mold wall contact. Also, silica sand exhibits anisotropic elastic behavior as will be discussed in the 
following sub-section which contributes to observing different $\varepsilon_{\mathrm{xx}}$ and $\varepsilon_{\mathrm{yy}}$ values. In all cases, $\varepsilon_{\mathrm{xx}}$ and $\varepsilon_{\mathrm{yy}}$ values were very small (less than $0.03 \%$ for Test 3 before particle fracture).

Figure 8 also shows the normal and shear strain components for the three sand particles of Test 3. The shear strain values sometimes exhibited inconsistent trend as the load increased or if there was a continuous increase or decrease in a particular direction up to the fracture point. All shear strain values for the top particle fluctuated throughout the test. On the other hand, a nonlinear continuous $\varepsilon_{\mathrm{xz}}$ and $\varepsilon_{\mathrm{yz}}$ were observed in the middle and bottom particles with the exception of a few data points. The $\varepsilon_{x y}$ curve varied in compression close to the zero strain line. Moreover, the shear strain values were about 15 folds smaller than the normal strain tensor components in all cases. Since shear strain is primarily influenced by the contact point orientation, loading mode, particle shape, and fabric, we did not expect a well-defined trend in shear strains as particles translated and rotated as the loading progressed, which caused particles to establish or lose contact with the mold walls. Elastic anisotropy might have contributed to such behavior.

Volumetric lattice strain $\left(\varepsilon_{\mathrm{v}}\right)$ of individual sand particles under the influence of compressive loading is depicted in Figure 9. $\varepsilon_{\mathrm{v}}$ was calculated as the summation of the three normal strains (i.e., $\varepsilon_{\mathrm{v}}=\varepsilon_{\mathrm{xx}}+\varepsilon_{\mathrm{yy}}+\varepsilon_{\mathrm{zz}}$ ). Figure 9 shows that the volume of silica sand particles decreased (i.e., particle volume decreased) in a nearly linear fashion as the compressive load increased up to particle fracture. This was expected since it represented the elastic volumetric strain.

The lattice axial strains $\left(\varepsilon_{\mathrm{zz}}\right)$ of all tracked particles of the three tests are plotted in Figure 10, which shows the results up to fracture of one of the particles in the sand column. All particles 
exhibited linear load versus strain responses with a small increase of the curve slope as the loading rate increased from $0.0125 \mathrm{~mm} / \mathrm{min}$ to $0.20 \mathrm{~mm} / \mathrm{min}$. The change of the slope with loading rate is in agreement with global load versus displacement measurements shown in Figure 3a, where higher loading rate caused an increase in the overall stiffness of the sand column.

Strain calculations for Tests 1 and 2 are summarized in Table 1. Compressive load (F) versus $\varepsilon_{\mathrm{zz}}$ measurements follow an almost perfect linear-elastic behavior up to fracture point with the exception of one particle (bottom particle of Test 1, see Table 1 and Figure 10) where we suspect that a partial fracture, which caused erratic behavior for loads higher than $14.75 \mathrm{~N}$, took place before complete disintegration. A linear regression fit function of $F$ versus $\varepsilon_{\mathrm{zz}}$ is also listed in the last column of Table 1 . A high correlation coefficient $\left(R^{2} \geq 0.993\right)$ was found between $F$ and $\varepsilon_{z z}$ for all particles except the bottom particle of Test 1, which demonstrates the accuracy of the measurements.

\section{Lattice strain-stress relationship}

The lattice strain and stress tensors are linearly related through the elastic constants which form a fourth-rank tensor. Because strain and stress tensors are symmetric, the full equation can be contracted to [39]:

$$
\sigma_{i}=c_{i j} \varepsilon_{j} \quad i, j=1 \ldots 6
$$

Here, the Voigt contraction was used. $c_{i j}$ is the elastic stiffness tensor. The components $c_{i j}^{c}$ are given in contracted notation and refer to a crystal frame orthonormal coordinate system $\{\widehat{\boldsymbol{x}}, \hat{\boldsymbol{y}}, \hat{\boldsymbol{z}}\}$. The values of $c_{i j}$ were obtained by expanding the $c_{i j}^{c}$ to a fourth-rank tensor, transforming it to the sample frame (the change of basis matrix given by $\mathbf{U}^{*} \mathbf{R}$ as described below), and back contracting. 
For the trigonal crystal system, $\widehat{\boldsymbol{x}}$ and $\hat{\mathbf{z}}$ are parallel to the $\mathbf{a}$ - and $\mathbf{c}$-axis, respectively. It is important to note that the $\mathbf{U}$-matrix in the Fable convention is defined as the change of basis matrix from an orthonormal coordinate system $\left\{\widehat{\boldsymbol{x}^{*}}, \widehat{\boldsymbol{y}^{*}}, \widehat{\mathbf{z}}^{*}\right\}$ attached to the reciprocal crystal frame $\left(\widehat{\boldsymbol{x}^{*}}, \widehat{\boldsymbol{z}}^{*}\right.$ parallel to $\mathbf{a}^{*}$ and $\mathbf{c}^{*}$, respectively) to the sample frame. Therefore, the change of basis matrix from the $\{\hat{\boldsymbol{x}}, \widehat{\boldsymbol{y}}, \hat{\boldsymbol{z}}\}$ system to the sample system is given by $\mathbf{U}^{*} \mathbf{R}$ where $\mathbf{R}$ is the change of coordinate matrix from the real $\{\widehat{\boldsymbol{x}}, \widehat{\boldsymbol{y}}, \hat{\boldsymbol{z}}\}$ to the reciprocal $\left\{\widehat{\boldsymbol{x}}^{*}, \widehat{\boldsymbol{y}}^{*}, \widehat{\mathbf{z}}^{*}\right\}$ crystal coordinate systems (a $30^{\circ}$ rotation around the c-axis). The stress tensors were then expanded from the contracted forms obtained using Equation 9 for which the experimental strain tensors were contracted to Voigt notation.

Figure 11a shows the evolution of the stress tensor components for the first seven applied load steps of the middle particle of Test 3 . We note first that quartz has a significant elastic anisotropy, as indicated by Young's moduli of $79.4 \mathrm{GPa}$ and $103 \mathrm{GPa}$ in the a- and c-axis directions, respectively, and a much larger directional variation of the Poisson ratio [39]. Thus anisotropic elasticity should be applied when converting lattice strains into stresses. The stress components showed a remarkably linear load dependence and uniaxial loading state over the first four applied loading steps. $\sigma_{z z}$ component exhibits a linear variation in stress-strain plot under uniaxial loading as expected. On the other hand, $\sigma_{x x}$ and $\sigma_{y y}$ components show compressional and tensional behavior under the effect of several factors including rotation, translation, boundary effect, and contact points. We concluded that the interactions of particles with the wall of the mold were initially small and that the experimental uncertainty on the stress components was on the order of a few MPa. Significant changes of the loading state observed during loading step five were attributed to a rearrangement of the particles. One now can easily generate the stressstrain plot for any particle using lattice strain measurements as input to calculate stress tensor. 
Figure $11 \mathrm{~b}$ shows an illustrative example for the stress-strain relationships for the normal strain components for the middle particle of Test 3. Shear stress-strain components were not plotted in Figure $11 \mathrm{~b}$ since they are very small when compared to normal stress-strain components.

\section{SUMMARY AND CONCLUSIONS}

3DXRD technique was successfully used to collect diffraction data and use those data to calculate in situ elastic lattice strain measurements of individual silica sand particles and track particle orientation under the effect of $1 \mathrm{D}$ compressive loading. The axial normal strain $\varepsilon_{\mathrm{zz}}$ exhibited a linear response in the range of 0 to $10^{-3}$ to the applied axial load between 0 and $30 \mathrm{~N}$ up to particle fracture. The relationship between $F$ and $\varepsilon_{z z}$ fits a linear regression model with a very high correlation coefficient $\left(\mathrm{R}^{2} \geq 0.993\right)$, which represents the linear elastic deformation of the sand particles. The volumetric strain decreased linearly with applied load up to particle fracture. An increase in the loading rate caused an increase in the slope of the load versus $\varepsilon_{\mathrm{zz}}$ relationships. The stress tensor was calculated using the elastic constants of quartz from literature and the lattice strain tensor. Experimental quantification of the evolution of particle orientation, lattice strain and stress tensors of silica sand provided valuable key measurements for the constitutive behavior of individual silica sand particles and can be used to develop, validate, or calibrate micromechanics-based finite element and discrete element methods to predict the constitutive behavior of granular materials.

\section{ACKNOWLEDGMENTS}

This material is based upon work supported by the National Science Foundation (NSF) under Grant No. (CMMI-1156436). The 3DXRD data were collected using the X-ray Operations and Research Beamline 1-ID and SMT scans were collected using the X-ray Operations and 
Research Beamline Station 13-BMD at the Advanced Photon Source (APS), Argonne National

Laboratory (ANL). We thank Dr. Mark Rivers of (APS) for help in performing the SMT scans.

We also acknowledge the support of GeoSoilEnviroCARS (Sector 13), which is supported by the

National Science Foundation - Earth Sciences (EAR-1128799), and the Department of Energy,

Geosciences (DE-FG02-94ER14466). Use of the Advanced Photon Source, an Office of Science

User Facility operated for the U.S. Department of Energy (DOE) Office of Science by Argonne

National Laboratory, was supported by the U.S. DOE under Contract No. DE-AC02-06CH11357

\section{REFERENCES}

1. Dafalias, Y.F.: Corotational Rates for Kinematic Hardening at Large Plastic-Deformations. J Appl Mech-T Asme 50(3), 561-565 (1983).

2. Manzari, M.T., Dafalias, Y.F.: A critical state two-surface plasticity model for sands. Geotechnique 47(2), 255-272 (1997).

3. Alsaleh, M.I., Voyiadjis, G.Z., Alshibli, K.A.: Modelling strain localization in granular materials using micropolar theory: Mathematical formulations. International Journal for Numerical and Analytical Methods in Geomechanics 30(15), 1501-1524 (2006).

4. De Borst, R.: Simulation of strain localization: a reappraisal of the Cosserat continuum. Engineering computations 8(4), 317-332 (1991).

5. Gudehus, G.: A comprehensive constitutive equation for granular materials. Journal of the Japanese Geotechnical Society : soils and foundation 36(1), 1-12 (1996).

6. Bardet, J.P., Proubet, J.: A Numerical Investigation of the Structure of Persistent Shear Bands in Granular Media. Geotechnique 41(4), 599-613 (1991).

7. Cundall, P.A.: Numerical experiments on localization in frictional materials. Archive of Applied Mechanics 59(2), 148-159 (1989). doi:10.1007/bf00538368

8. Oda, M., Kazama, H.: Microstructure of shear bands and its relation to the mechanisms of dilatancy and failure of dense granular soils. Geotechnique 48(4), 465-481 (1998).

9. Regueiro, R.A., Yan, B.: Concurrent Multiscale Computational Modeling for Dense Dry Granular Materials Interfacing Deformable Solid Bodies. In: Wan, R., Alsaleh, M., Labuz, J. (eds.) Bifurcations, Instabilities and Degradations in Geomaterials, vol. 0. Springer Series in Geomechanics and Geoengineering, pp. 251-273. Springer Berlin Heidelberg, (2011)

10. Calvetti, F., Combe, G., Lanier, J.: Experimental micromechanical analysis of a 2D granular material: relation between structure evolution and loading path. Mechanics of Cohesivefrictional Materials 2(2), 121-163 (1997). 
11. Oda, M., Konishi, J., Nemat-Nasser, S.: Experimental Micromechanical Evaluation of Strength of Granular Materials: Effects of Particle Rolling. Mech Mater 1, 269-283 (1982).

12. Rowe, P.W.: The Stress-Dilatancy Relation for Static Equilibrium of an Assembly of Particles in Contact. Proceedings of the Royal Society of London. Series A. Mathematical and Physical Sciences 269(1339), 500-527 (1962).

13. Allersma, H.G.B.: Photo-elastic Stress Analysis and Rate of Strain on the Measured In Situ Shear Strength of Soils. In: IUTAM Conference on Deformation and Failure of Granular Materials 1982, pp. 345-353. Balkema

14. Majmudar, T.S., Behringer, R.P.: Contact force measurements and stress-induced anisotropy in granular materials. Nature 435(1079), 1079-1082 (2005).

15. Geng, J.F., Reydellet, G., Clement, E., Behringer, R.P.: Green's function measurements of force transmission in 2D granular materials. Physica D 182(3-4), 274-303 (2003).

16. vanDoorn, E., Behringer, R.P.: Dilation of a vibrated granular layer. Europhys Lett 40(4), 387-392 (1997).

17. Alshibli, K.A., Reed, A.: Advances in Computed Tomography for Geomaterials: Geox2010. Wiley, Hoboken, NJ, USA (2010)

18. Hasan, A., Alshibli, K.: Three dimensional fabric evolution of sheared sand. Granular Matter 14(4), 469-482 (2012).

19. Butler, L.G.: Progress Towards Neutron Tomography at the US Spallation Neutron Source. In: Alshibli, K.A., Reed, A.H. (eds.) Advances in Computed Tomography for Geomaterials. pp. 366-373. John Wiley \& Sons, Inc., (2010)

20. Penumadu, D., Dutta, A., Luo, X., Thomas, K.: Nano and neutron science applications for geomechanics. KSCE J Civ Eng 13(4), 233-242 (2009).

21. Frischbutter, A., Neov, D., Scheffzuk, C., Vrana, M., Walther, K.: Lattice strain measurements on sandstones under load using neutron diffraction. J Struct Geol 22(1112), 1587-1600 (2000).

22. Darling, T.W., TenCate, J.A., Brown, D.W., Clausen, B., Vogel, S.C.: Neutron diffraction study of the contribution of grain contacts to nonlinear stress-strain behavior. Geophys Res Lett 31(16), L16604 (2004).

23. Lienert, U., Schulze, C., Honkimaki, V., Tschentscher, T., Garbe, S., Hignette, O., Horsewell, A., Lingham, M., Poulsen, H.F., Thomsen, N.B., Ziegler, E.: Focusing optics for high-energy X-ray diffraction. J Synchrotron Radiat 5, 226-231 (1998).

24. Oddershede, J., Schmidt, S., Poulsen, H.F., Sorensen, H.O., Wright, J., Reimers, W.: Determining grain resolved stresses in polycrystalline materials using three-dimensional X-ray diffraction. Journal of Applied Crystallography 43(3), 539-549 (2010).

25. Poulsen, H.F.: Three-dimensional X-ray diffraction microscopy: mapping polycrystals and their dynamics, vol. 205. Springer, (2004)

26. Poulsen, H.F., Margulies, L., Schmidt, S., Winther, G.: Lattice rotations of individual bulk grains: Part I: 3D X-ray characterization. Acta Mater 51(13), 3821-3830 (2003). 
27. Poulsen, H.F., Nielsen, S.F., Lauridsen, E.M., Schmidt, S., Suter, R.M., Lienert, U., Margulies, L., Lorentzen, T., Juul Jensen, D.: Three-dimensional maps of grain boundaries and the stress state of individual grains in polycrystals and powders. Journal of Applied Crystallography 34(6), 751-756 (2001).

28. Jensen, D.J., Offerman, S.E., Sietsma, J.: 3DXRD characterization and modeling of solidstate transformation processes. Mrs Bull 33(6), 621-629 (2008).

29. Martins, R.V., Margulies, L., Schmidt, S., Poulsen, H.F., Leffers, T.: Simultaneous measurement of the strain tensor of 10 individual grains embedded in an Al tensile sample. Mat Sci Eng a-Struct 387, 84-88 (2004).

30. Hall, S., Wright, J., Pirling, T., Andò, E., Hughes, D., Viggiani, G.: Can intergranular force transmission be identified in sand? Granular Matter 13(3), 251-254 (2011).

31. Fable. http://sourceforge.net/apps/trac/fable/wiki (2011).

32. Kenesei, P.: DIGIgrain. http://sourceforge.net/apps/trac/digigrain/wiki (2012).

33. Levien, L., Prewitt, C.T., Weidner, D.J.: Structure and Elastic Properties of Quartz at Pressure. Am Mineral 65(9-10), 920-930 (1980).

34. Wright, J.: ImageD11. http://sourceforge.net/apps/trac/fable/wiki/imaged11 (2005).

35 .

Schmidt, S.:

GrainSpotter

$\mathrm{v}$.

0.82 . http://fable.svn.sourceforge.net/svnroot/fable/GrainSpotter (2010).

36. Morawiec, A., Field, D.P.: Rodrigues parameterization for orientation and misorientation distributions. Philosophical Magazine A 73(4), 1113-1130 (1996).

37. Margulies, L., Lorentzen, T., Poulsen, H.F., Leffers, T.: Strain tensor development in a single grain in the bulk of a polycrystal under loading. Acta Mater 50(7), 1771-1779 (2002).

38. Ludwig, W., Reischig, P., King, A., Herbig, M., Lauridsen, E.M., Johnson, G., Marrow, T.J., Buffiere, J.Y.: Three-dimensional grain mapping by $\mathrm{x}$-ray diffraction contrast tomography and the use of Friedel pairs in diffraction data analysis. Review of Scientific Instruments 80(3), 033905-033909 (2009).

39. Heyliger, P., Ledbetter, H., Kim, S.: Elastic constants of natural quartz. J Acoust Soc Am 114(2), 644-650 (2003). 
Table 1. Elastic lattice strain values for Tests 1 and 2 and associated statistical fit function for $\varepsilon_{\mathrm{zz}}$

\begin{tabular}{|c|c|c|c|c|c|c|c|c|c|}
\hline Test & Particle & $\begin{array}{l}\text { Load, F } \\
\text { (N) }\end{array}$ & $\begin{array}{l}\varepsilon_{\mathrm{xx}} \\
(\%)\end{array}$ & $\begin{array}{l}\varepsilon_{\mathrm{yy}} \\
(\%)\end{array}$ & $\begin{array}{c}\varepsilon_{\mathrm{zZ}} \\
(\%)\end{array}$ & $\begin{array}{c}\varepsilon_{\mathrm{xy}} \\
(\%)\end{array}$ & $\begin{array}{l}\varepsilon_{\mathrm{xz}} \\
(\%)\end{array}$ & $\begin{array}{c}\varepsilon_{\mathrm{yz}} \\
(\%)\end{array}$ & Fit function for $\varepsilon_{\mathrm{zz}}$ \\
\hline \multirow{15}{*}{1} & \multirow{5}{*}{ Bottom* } & 0 & 0 & 0 & 0 & 0 & 0 & 0 & \multirow{5}{*}{$\begin{array}{l}F=820.290 \varepsilon_{z z} \\
R^{2}=0.800\end{array}$} \\
\hline & & 14.75 & -0.0021 & -0.0036 & 0.0383 & -0.0038 & 0.0028 & -0.0018 & \\
\hline & & 19.75 & 0.0074 & 0.0000 & 0.0160 & 0.0046 & 0.0035 & 0.0064 & \\
\hline & & 28.5 & 0.0155 & -0.0004 & 0.0259 & 0.0093 & 0.0053 & 0.0102 & \\
\hline & & 35.25 & 0.0080 & 0.0026 & 0.0277 & 0.0070 & 0.0052 & 0.0086 & \\
\hline & \multirow{5}{*}{ Middle } & 0 & 0.0000 & 0.0000 & 0.0000 & 0.0000 & 0.0000 & 0.0000 & \multirow{5}{*}{$\begin{array}{l}\mathrm{F}=333.856 \quad \varepsilon_{\mathrm{zz}} \\
\mathrm{R}^{2}=0.993\end{array}$} \\
\hline & & 14.75 & 0.0059 & 0.0013 & 0.0383 & 0.0024 & -0.0049 & -0.0054 & \\
\hline & & 19.75 & 0.0119 & -0.0004 & 0.0510 & 0.0040 & -0.0094 & -0.0066 & \\
\hline & & 28.5 & 0.0113 & -0.0037 & 0.0837 & 0.0087 & -0.0103 & -0.0056 & \\
\hline & & 35.25 & 0.0109 & -0.0131 & 0.1125 & 0.0120 & -0.0163 & -0.0095 & \\
\hline & \multirow{5}{*}{ Top } & 0 & 0.0000 & 0.0000 & 0.0000 & 0.0000 & 0.0000 & 0.0000 & \multirow{5}{*}{$\begin{array}{l}\mathrm{F}=416.469 \quad \varepsilon_{z z} \\
\mathrm{R}^{2}=0.999\end{array}$} \\
\hline & & 14.75 & 0.0088 & -0.0039 & 0.0370 & -0.0090 & -0.0167 & -0.0033 & \\
\hline & & 19.75 & 0.0106 & -0.0069 & 0.0475 & -0.0112 & -0.0193 & -0.0059 & \\
\hline & & 28.5 & 0.0098 & -0.0125 & 0.0697 & -0.0115 & -0.0238 & -0.0085 & \\
\hline & & 35.25 & 0.0075 & -0.0099 & 0.0828 & -0.0111 & -0.0276 & -0.0053 & \\
\hline \multirow{12}{*}{2} & \multirow{4}{*}{ Bottom } & 0 & 0.0000 & 0.0000 & 0.0000 & 0.0000 & 0.0000 & 0.0000 & \multirow{4}{*}{$\begin{array}{l}\mathrm{F}=340.439 \quad \varepsilon_{\mathrm{zz}} \\
\mathrm{R}^{2}=0.998\end{array}$} \\
\hline & & 10.75 & -0.0064 & -0.0021 & 0.0308 & -0.0057 & -0.0023 & 0.0043 & \\
\hline & & 18.75 & -0.0123 & -0.0068 & 0.0590 & -0.0091 & 0.0049 & 0.0027 & \\
\hline & & 27.85 & -0.0147 & -0.0110 & 0.0792 & -0.0122 & 0.0023 & 0.0039 & \\
\hline & \multirow{4}{*}{ Middle } & 0 & 0.0000 & 0.0000 & 0.0000 & 0.0000 & 0.0000 & 0.0000 & \multirow{4}{*}{$\begin{array}{l}\mathrm{F}=357.058 \varepsilon_{\mathrm{zz}} \\
\mathrm{R}^{2}=0.995\end{array}$} \\
\hline & & 10.75 & -0.0044 & 0.0017 & 0.0263 & -0.0017 & 0.0105 & 0.0032 & \\
\hline & & 18.75 & -0.0056 & -0.0052 & 0.0483 & -0.0011 & 0.0099 & 0.0045 & \\
\hline & & 27.85 & -0.0160 & -0.0071 & 0.0817 & 0.0053 & 0.0169 & 0.0066 & \\
\hline & \multirow{4}{*}{ Top } & 0 & 0.0000 & 0.0000 & 0.0000 & 0.0000 & 0.0000 & 0.0000 & \multirow{4}{*}{$\begin{array}{l}\mathrm{F}=366.443 \quad \varepsilon_{\mathrm{zz}} \\
\mathrm{R}^{2}=0.997\end{array}$} \\
\hline & & 10.75 & 0.0021 & -0.0012 & 0.0249 & -0.0005 & 0.0006 & -0.0052 & \\
\hline & & 18.75 & -0.0122 & -0.0003 & 0.0496 & 0.0007 & -0.0019 & -0.0078 & \\
\hline & & 27.85 & -0.0175 & -0.0051 & 0.0784 & 0.0004 & 0.0005 & -0.0095 & \\
\hline
\end{tabular}

* Particle fractured at small load $(14.75 \mathrm{~N})$. 


\section{LIST OF FIGURES}

Figure 1. Schematic illustration of (a) Bragg diffraction; and (b) far field 3DXRD measurement geometry

Figure 2. Photo and schematic of 1D compression test cell

Figure 3. (a) Load versus displacement relationship of the three 1D compression experiments. Only top load cell measurements are plotted. (b) SMT images of a similar specimen. Compression is positive

Figure 4. The translation of the diffraction spots as axial load increases. The spots begin to move along the azimuthal direction $\eta$ with increasing load in different directions. Diffraction spots broaden in two directions and new reflections appear with increasing load due to new fragments generated after particle fracture. Also, diffraction spots move along the azimuthal direction $\eta$ on the detector plane and broadened in two directions due to particle orientation change and deformation

Figure 5. Example $\eta-\omega$ maps of the center layer of the sand column of Test 3 for selected load steps

Figure 6. Visual comparison of the reflection spots that were measured and predicted by GrainSpotter for a reference particle for load steps 0 to 2

Figure 7. (a) The variation of misorientation angle and $\Delta$ orientation for the middle particle of Test 3 at consecutive loads. (b) The orientation trajectory of the middle particle of Test 3 in Rodrigues space representation. 1/8 octant of the fundamental zone (red lines) is shown. The coordinates refer to the sample system as defined in Figure 1b. The blue circles indicate particle orientations at different loads (the initial orientation is marked by a solid green circle). The orientation trajectory is indicated by the solid lines connecting the load-dependent orientation vectors. The projections of the orientation trajectory onto the $\mathrm{xy}, \mathrm{yz}$, and $\mathrm{xz}$ sample planes are also shown.

Figure 8. Evolution of lattice strain measurements as a function of axial load for Test 3 for (a) top particle, (b) middle particle, and (c) bottom particle

Figure 9. Volumetric strain versus load for (a) top particle, (b) middle particle, and (c) bottom particle of Test 3

Figure 10. Axial strain values $\left(\varepsilon_{\mathrm{zz}}\right)$ for all particles up to first particle fracture point at different loading rates

Figure 11. (a) Applied load versus sample frame stress tensor components of the middle particle of Test 3 , and (b) stress versus strain variation for the middle particle of Test 3 . 


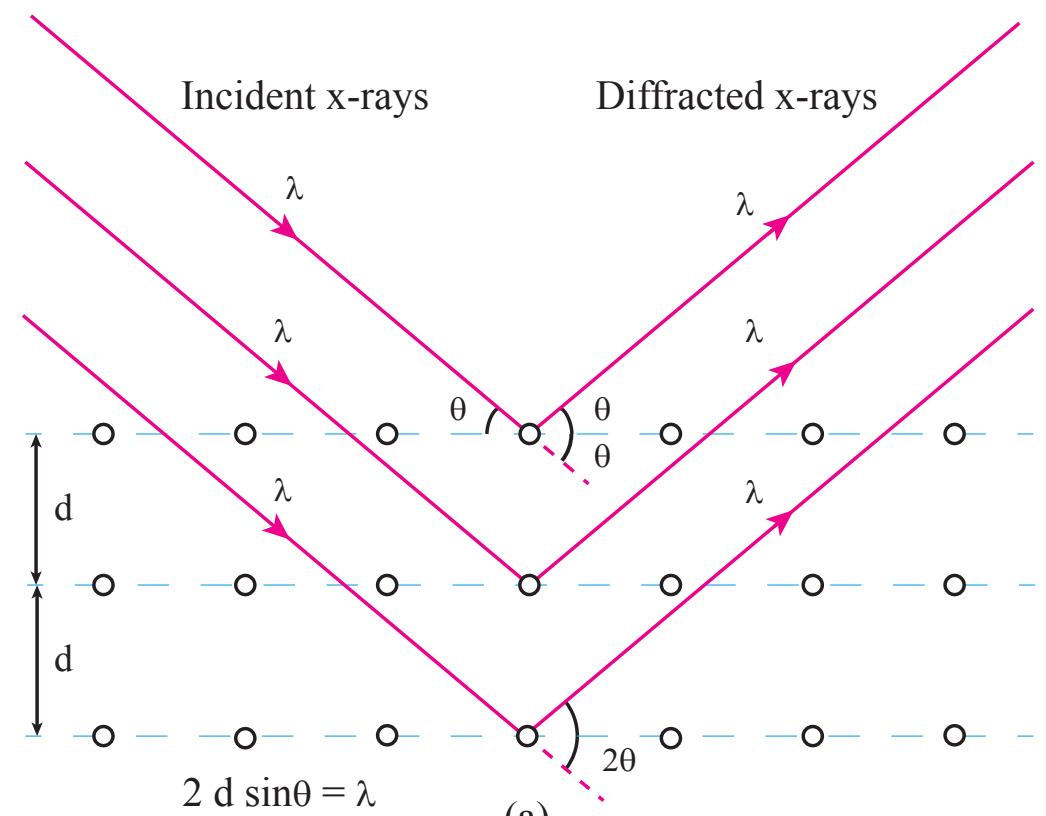

(a)

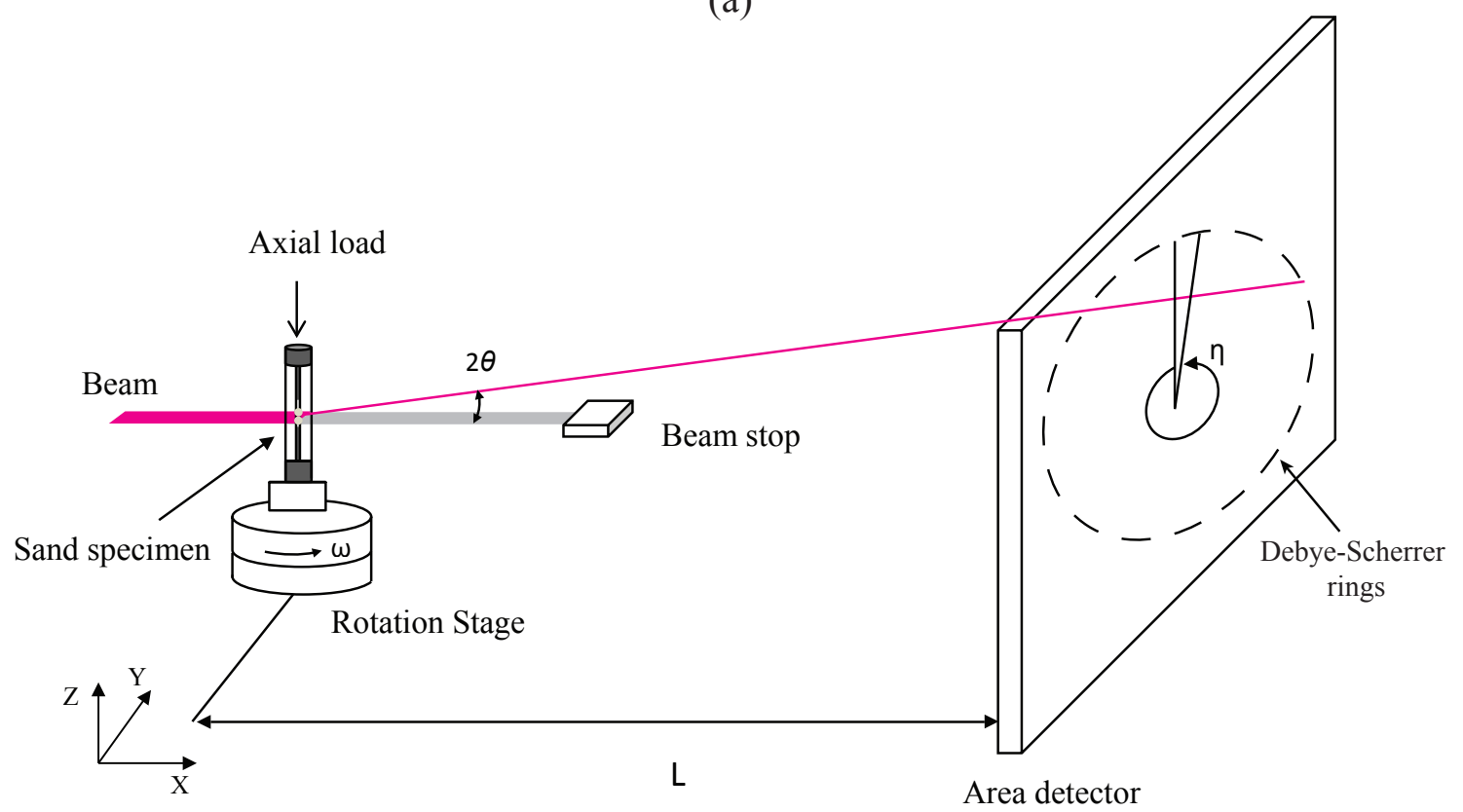

(b)

Figure 1. Schematic illustration of (a) Bragg diffraction; and (b) far field 3DXRD measurement geometry 

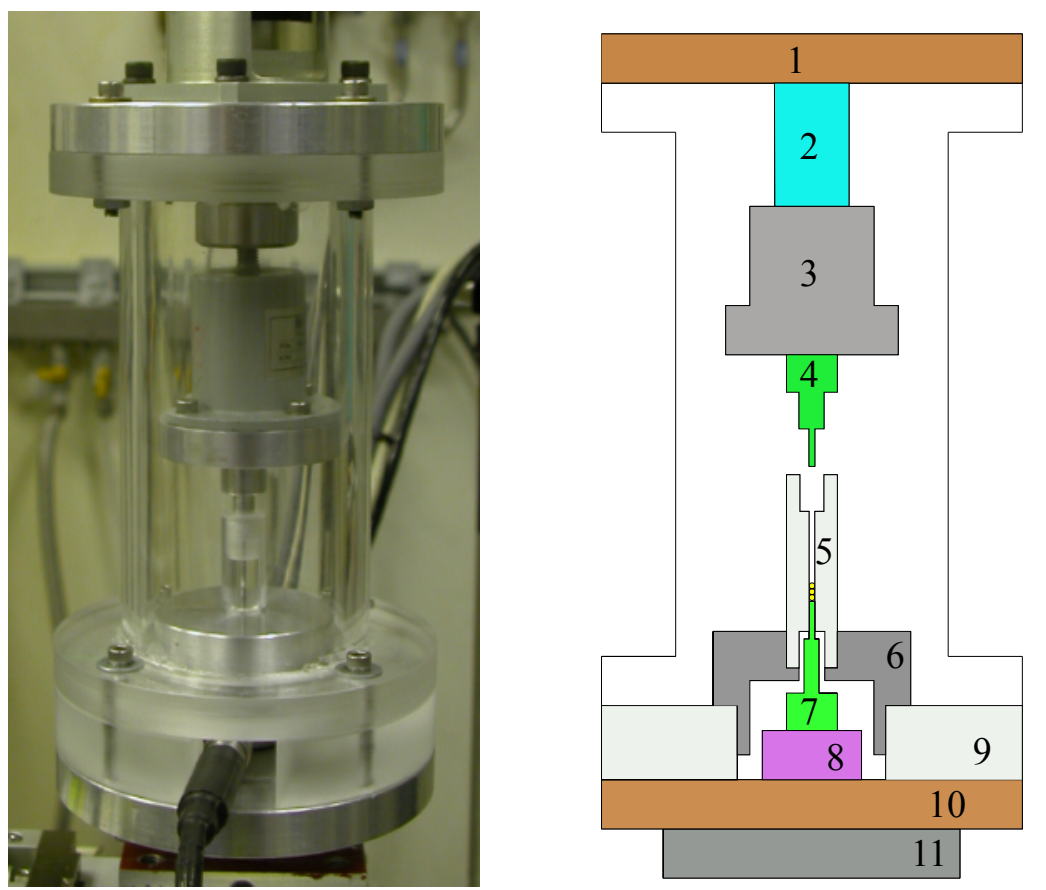

1: Top plate

2: Stepper motor to apply axial load

3: Top load cell

4: Top loading plate

5: Acrylic mold with sand particles

6: Aluminum spacer

7: Bottom loading plate

8: Bottom load cell

9: Acrylic spacer

10: Bottom plate

11: Cell-stage connection plate

Figure 2. Photo and schematic of 1D compression test cell 


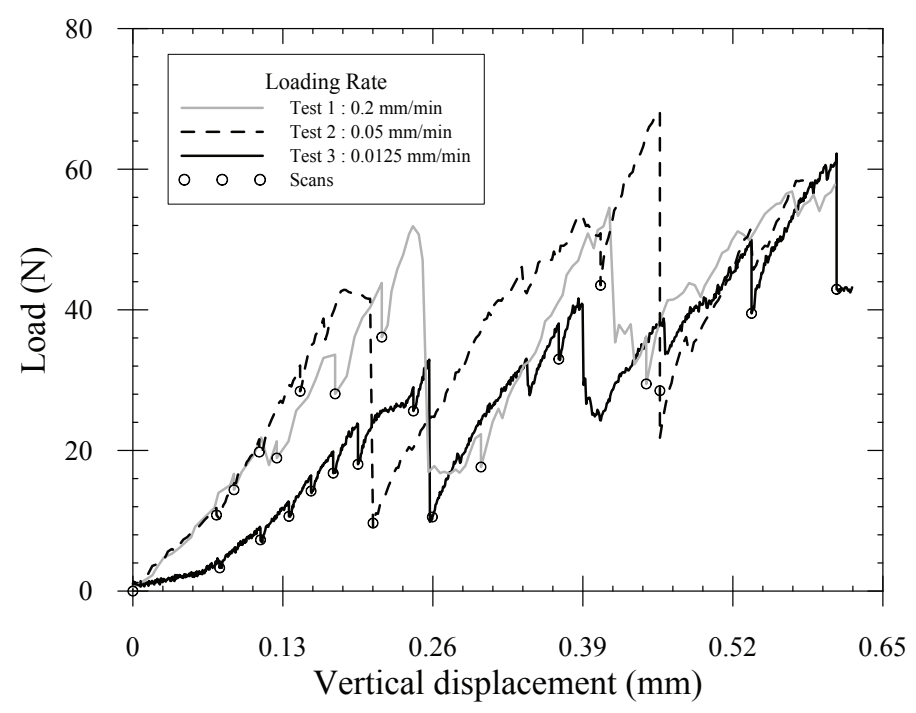

(a)

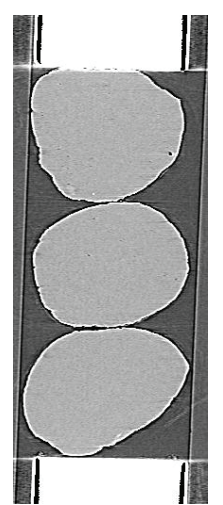

$\Delta_{\mathrm{Z}}=0 \mu$ Load $=0 \mathrm{~N}$

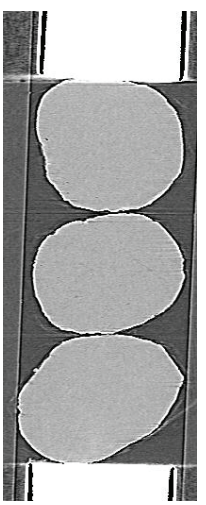

$\Delta_{\mathrm{Z}}=54.5 \mu$ $\operatorname{Load}=30 \mathrm{~N}$

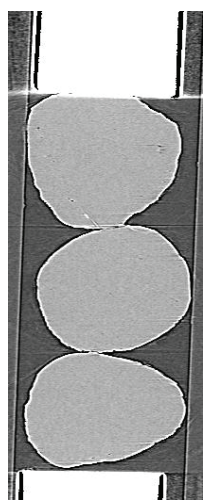

$\Delta_{\mathrm{Z}}=79.2 \mu$ $\operatorname{Load}=48 \mathrm{~N}$

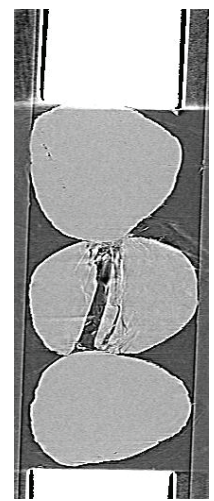

$\Delta_{\mathrm{Z}}=148.5 \mu$ Load $=22 \mathrm{~N}$

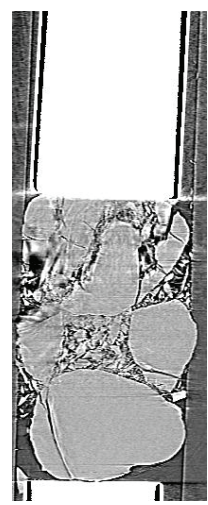

$\Delta_{\mathrm{Z}}=560 \mu$ Load $=88 \mathrm{~N}$

Figure 3. (a) Load versus displacement relationship of the three 1D compression experiments. Only top load cell measurements are plotted. (b) SMT images of a similar specimen. Compression is positive 


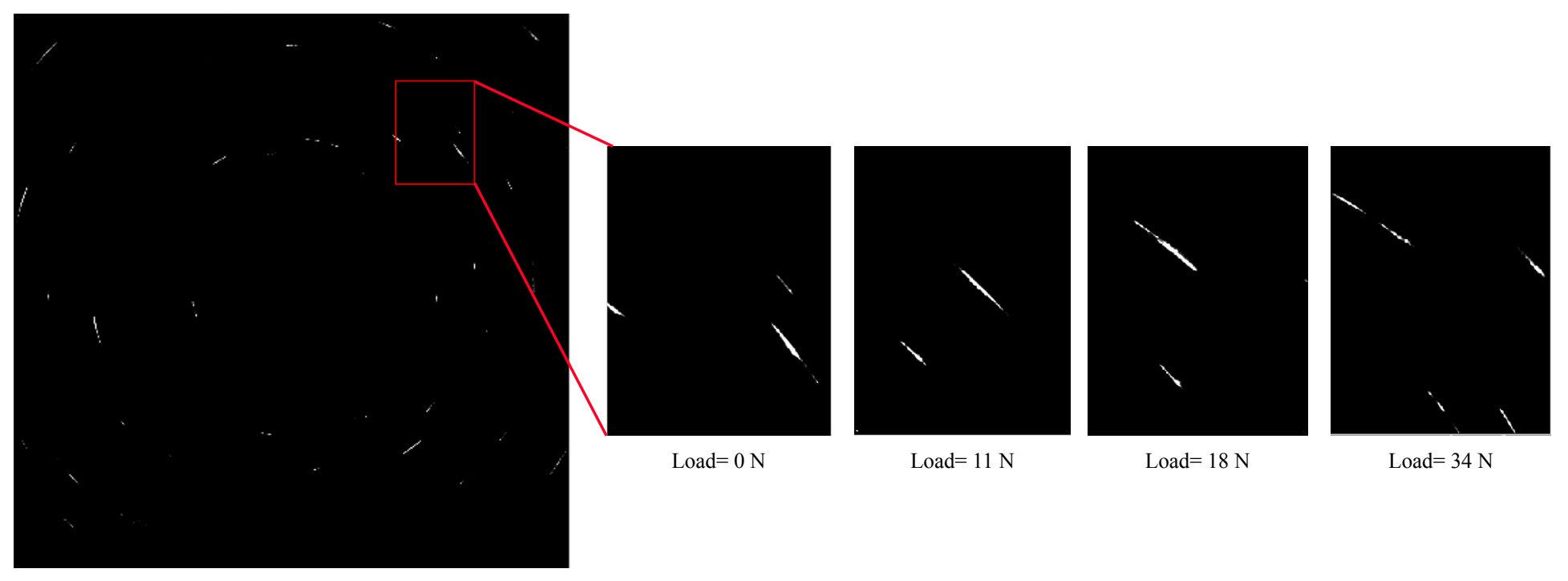

Figure 4. The translation of the diffraction spots as axial load increases. The spots begin to move along the azimuthal direction $\eta$ with increasing load in different directions. Diffraction spots broaden in two directions and new reflections appear with increasing load due to new fragments generated after particle fracture. Also, diffraction spots move along the azimuthal direction $\eta$ on the detector plane and broadened in two directions due to particle orientation change and deformation 


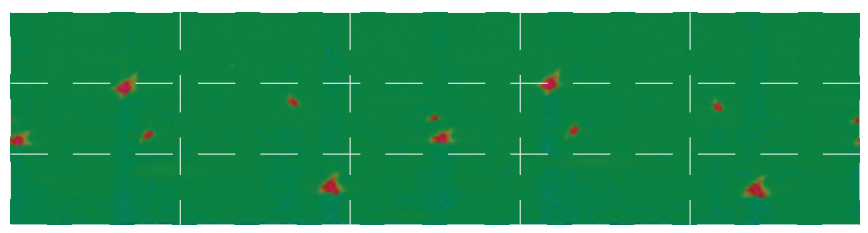

Scan \# 1 Load $=0 \mathrm{~N}$

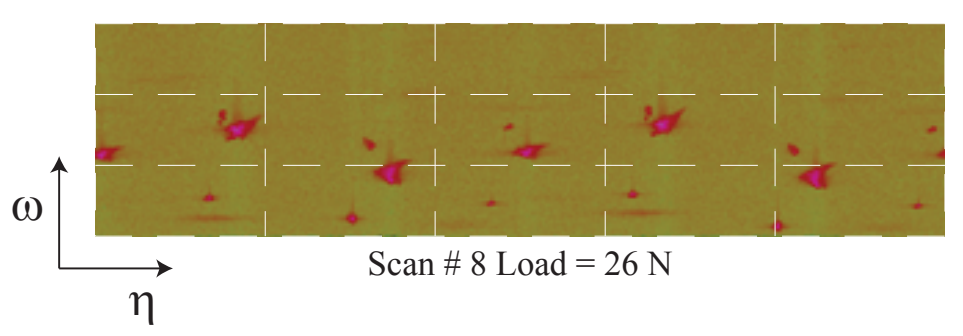

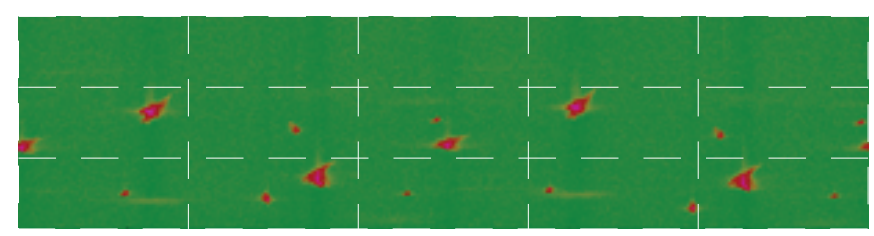

Scan \# 7 Load $=18 \mathrm{~N}$

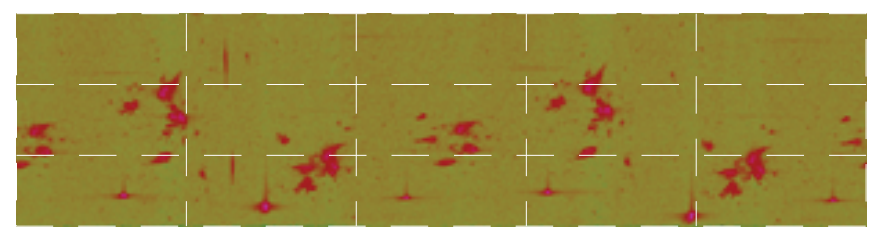

Scan \# $10 \mathrm{Load}=34 \mathrm{~N}$

Figure 5. Example $\eta-\omega$ maps of the center layer of the sand column of Test 3 for selected load steps 

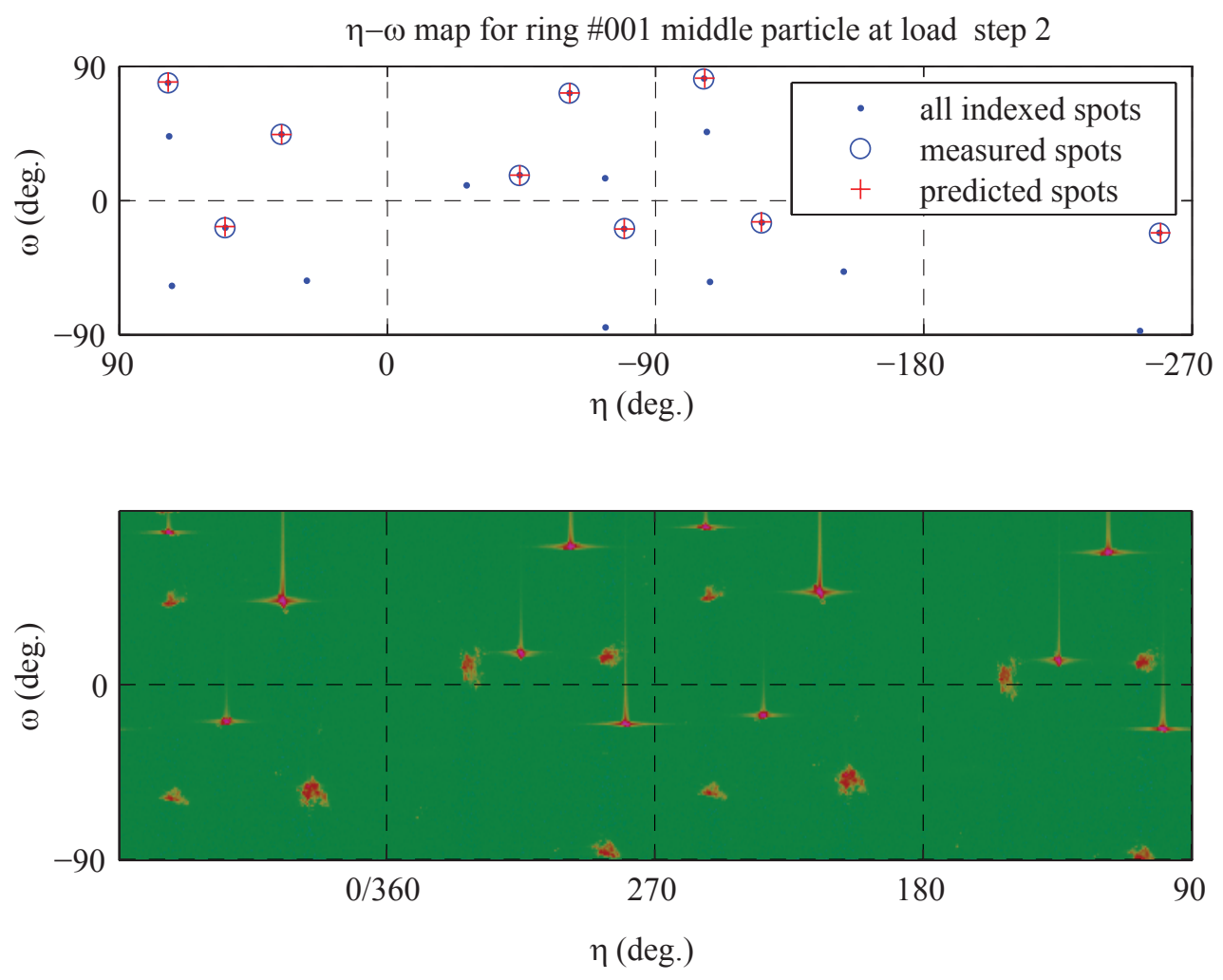

Figure 6. Visual comparison of the reflection spots that were measured and predicted by GrainSpotter for a reference particle for load steps 0 to 2 


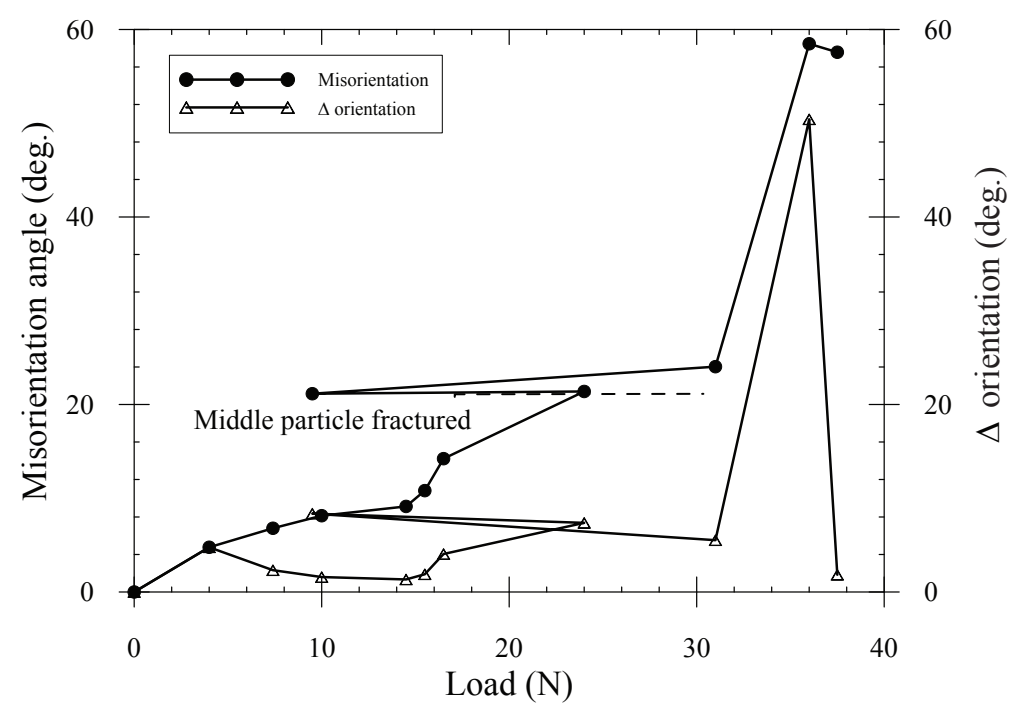

(a)

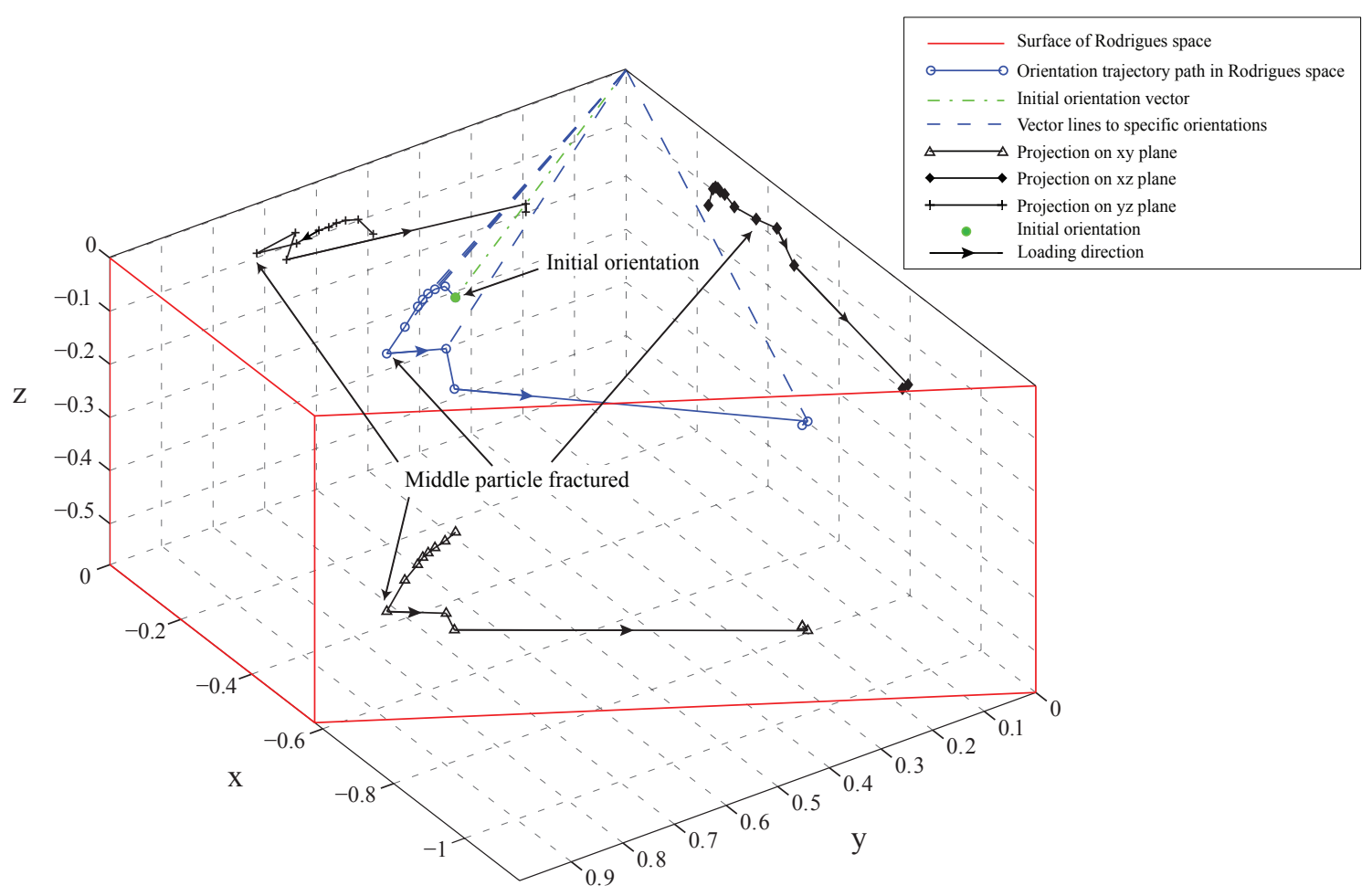

(b)

Figure 7. (a) The variation of misorientation angle and $\Delta$ orientation for the middle particle of Test 3 at consecutive loads. (b) The orientation trajectory of the middle particle of Test 3 in Rodrigues space representation. 1/8 octant of the fundamental zone (red lines) is shown. The coordinates refer to the sample system as defined in Figure $1 \mathrm{~b}$. The blue circles indicate particle orientations at different loads (the initial orientation is marked by a solid green circle). The orientation trajectory is indicated by the solid lines connecting the load-dependent orientation vectors. The projections of the orientation trajectory onto the $\mathrm{xy}, \mathrm{yz}$, and $\mathrm{xz}$ sample planes are also shown. 


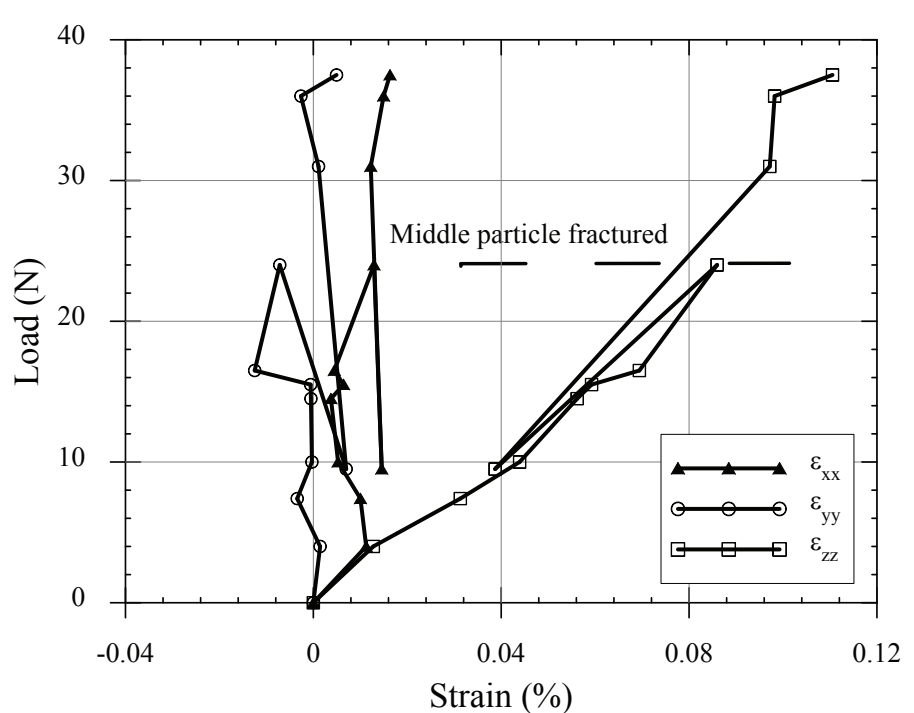

(a)

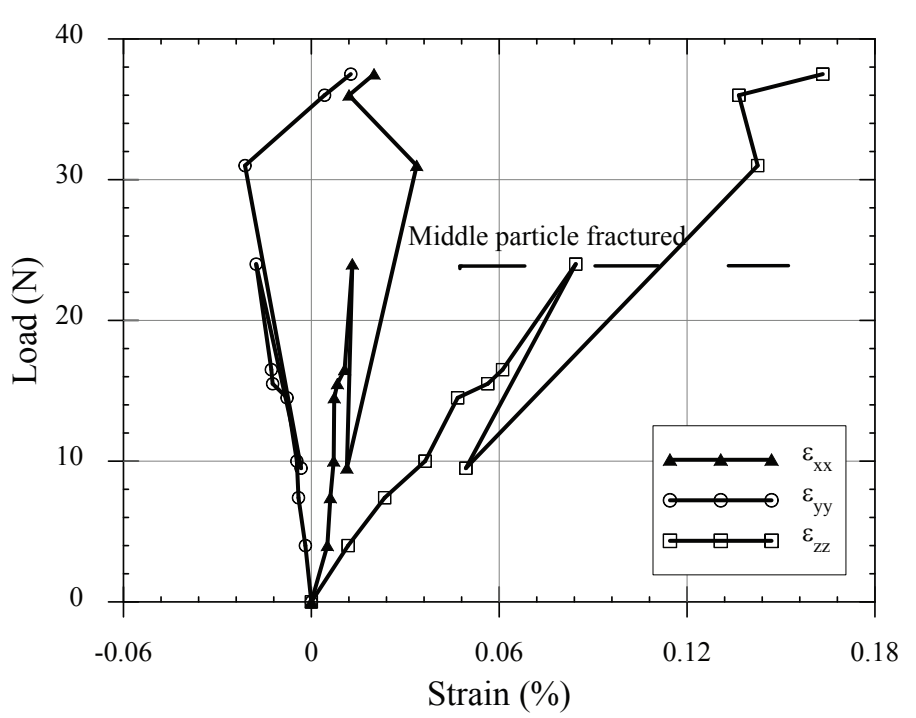

(b)

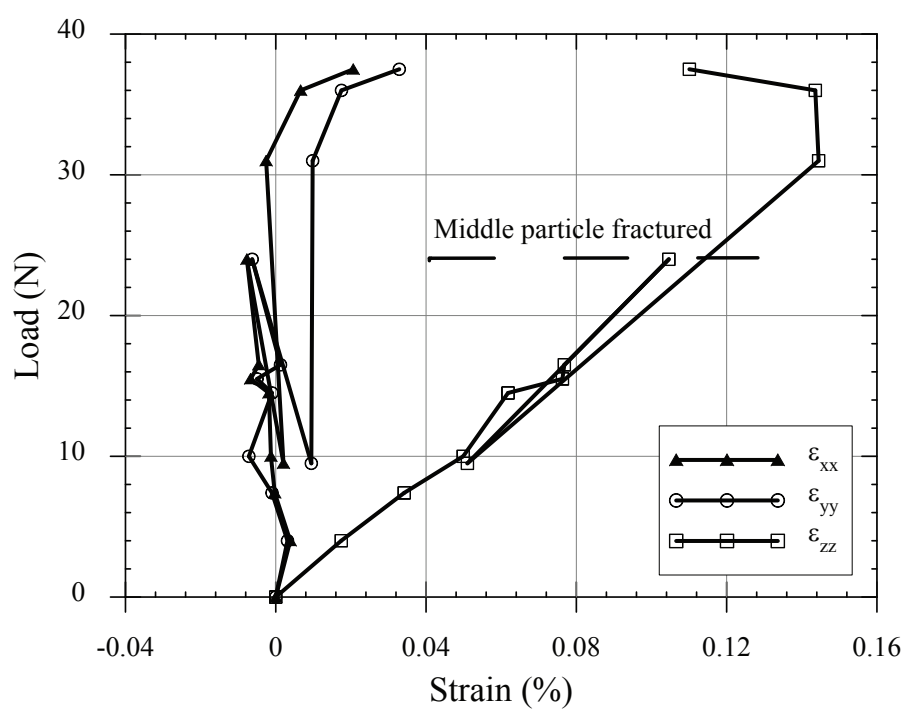

(c)

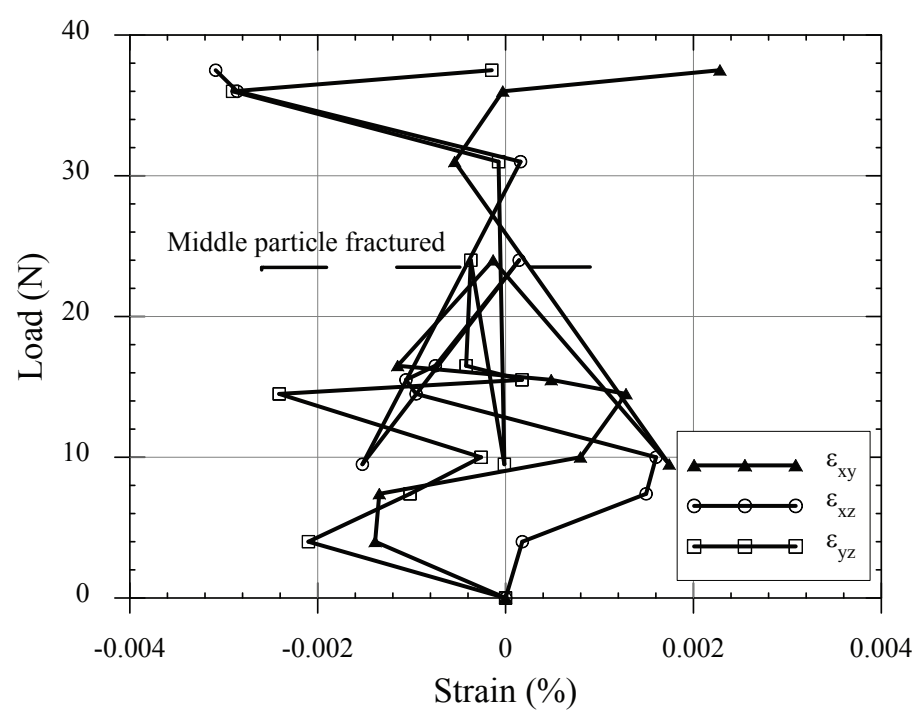

(a)

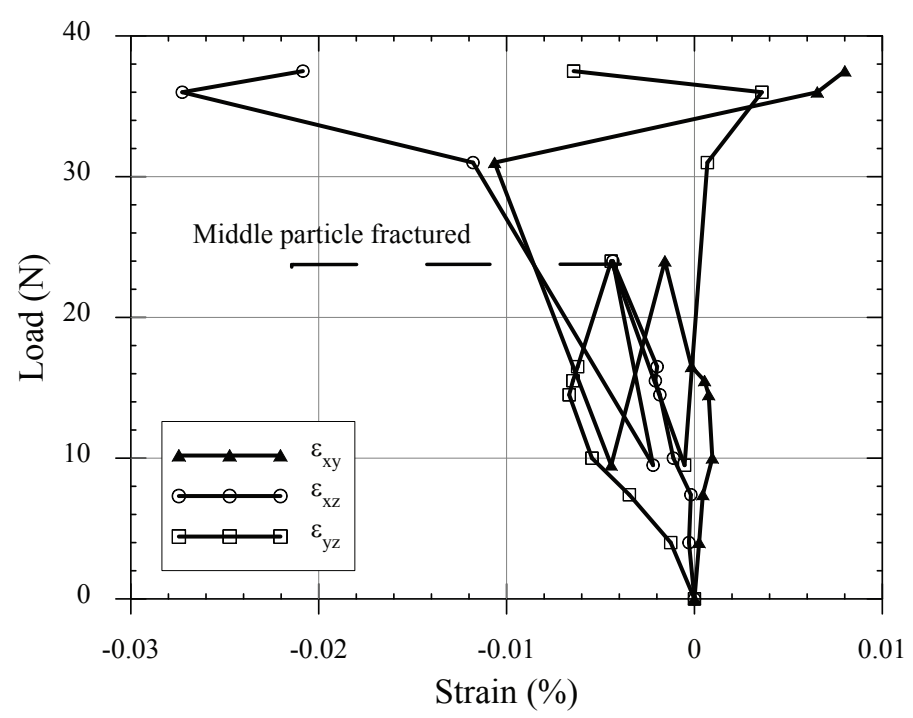

(b)

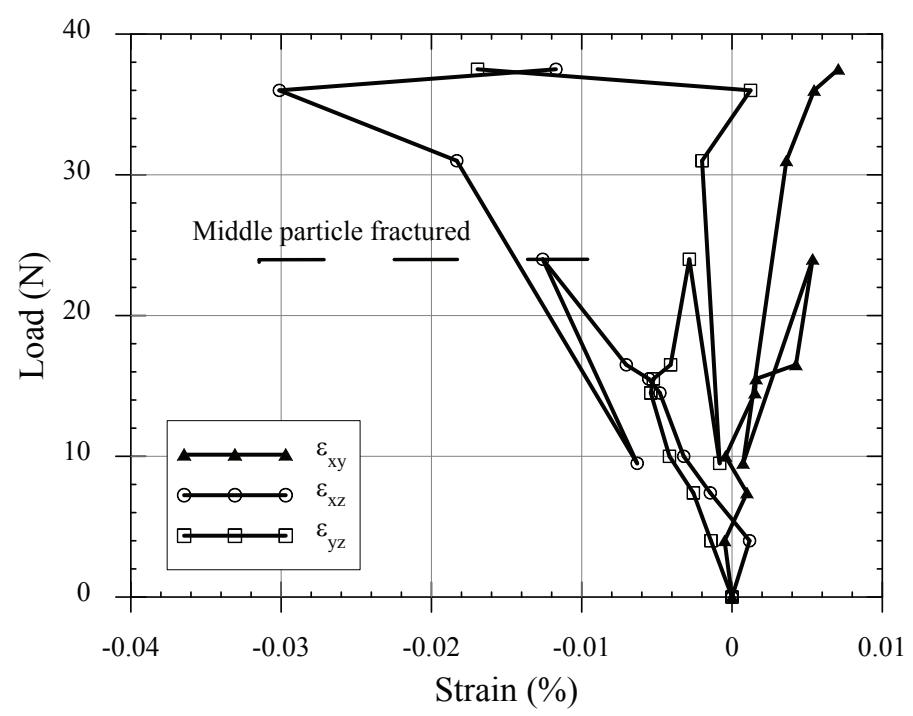

(c)

Figure 8. Evolution of lattice strain measurements as a function of axial load for Test 3 for (a) top particle, (b) middle particle, and (c) bottom particle 


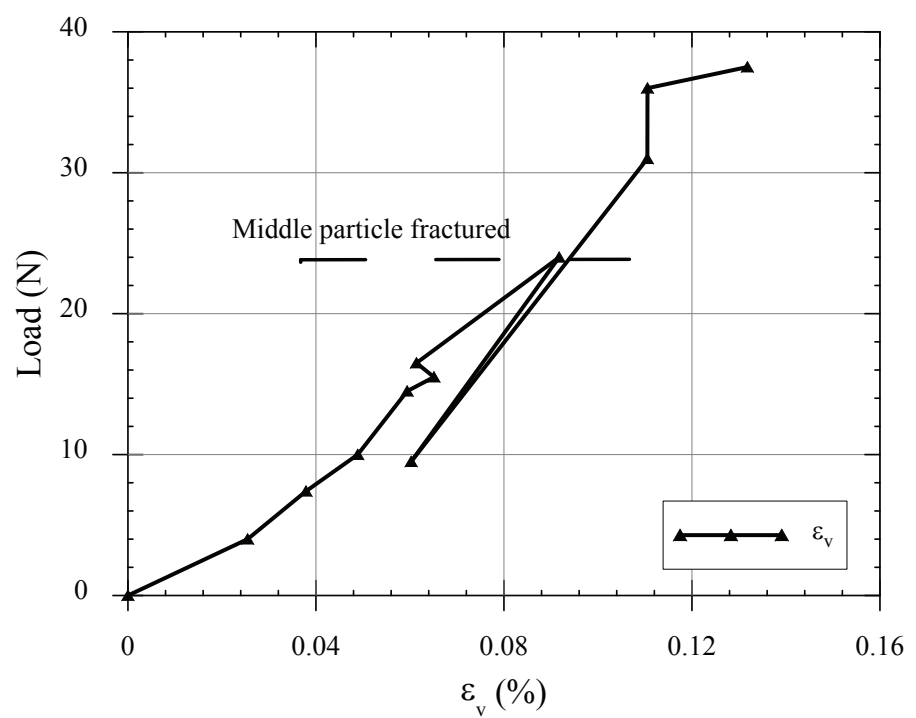

(a)

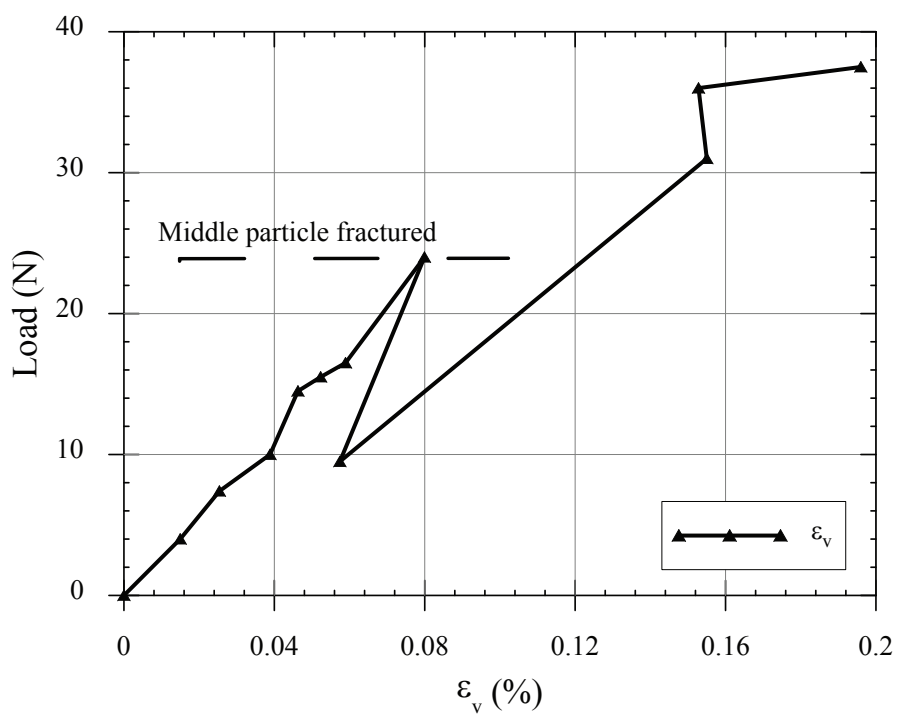

(b)

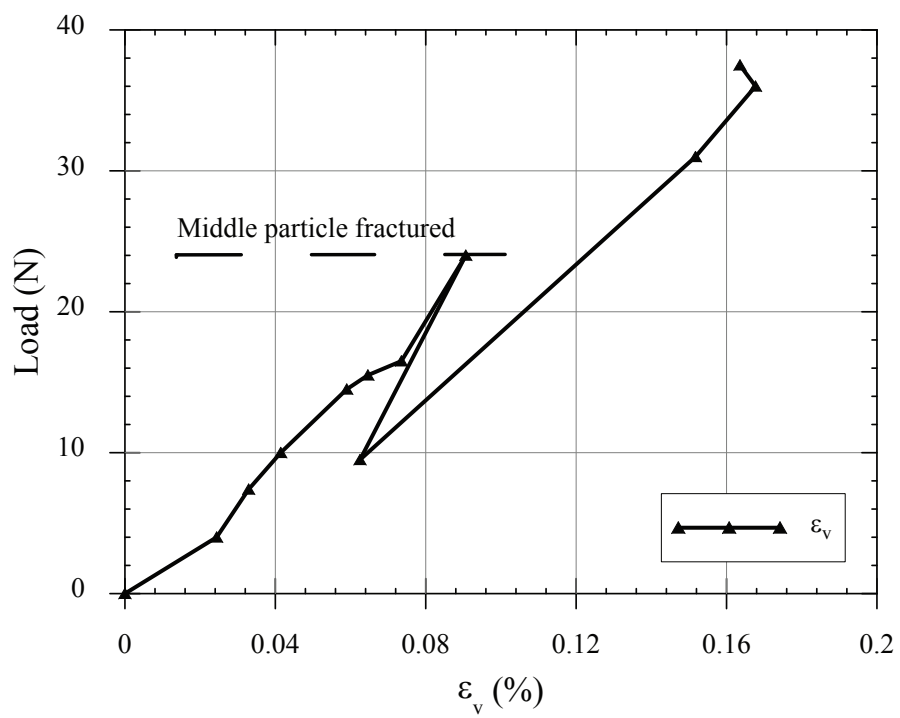

(c)

Figure 9. Volumetric strain versus load for (a) top particle, (b) middle particle, and (c) bottom particle of Test 3 


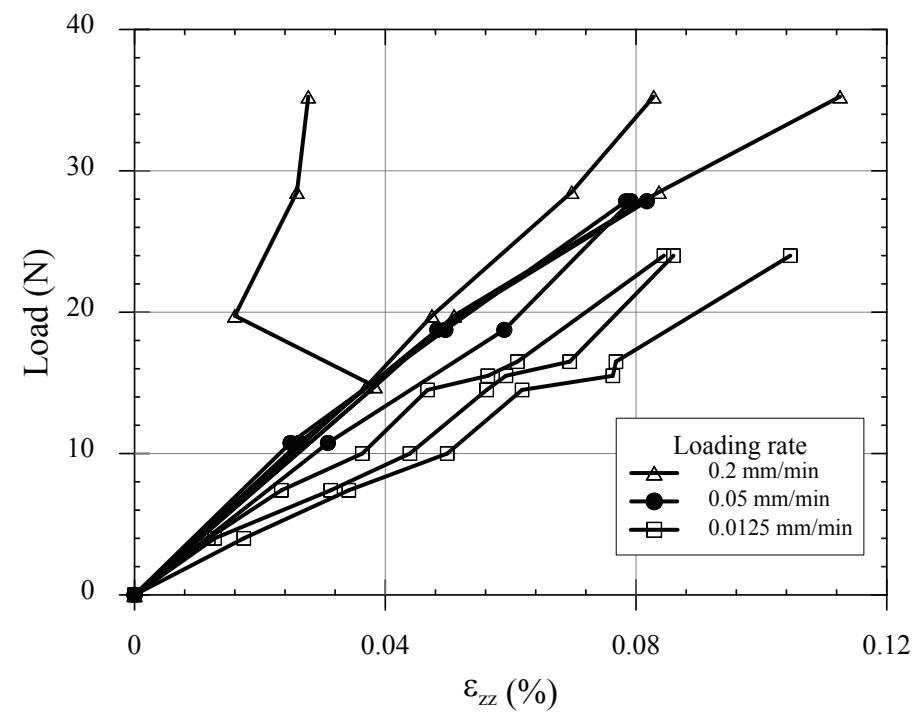

Figure 10. Axial strain values $\left(\varepsilon_{\mathrm{zZ}}\right)$ for all particles up to first particle fracture point at different loading rates 


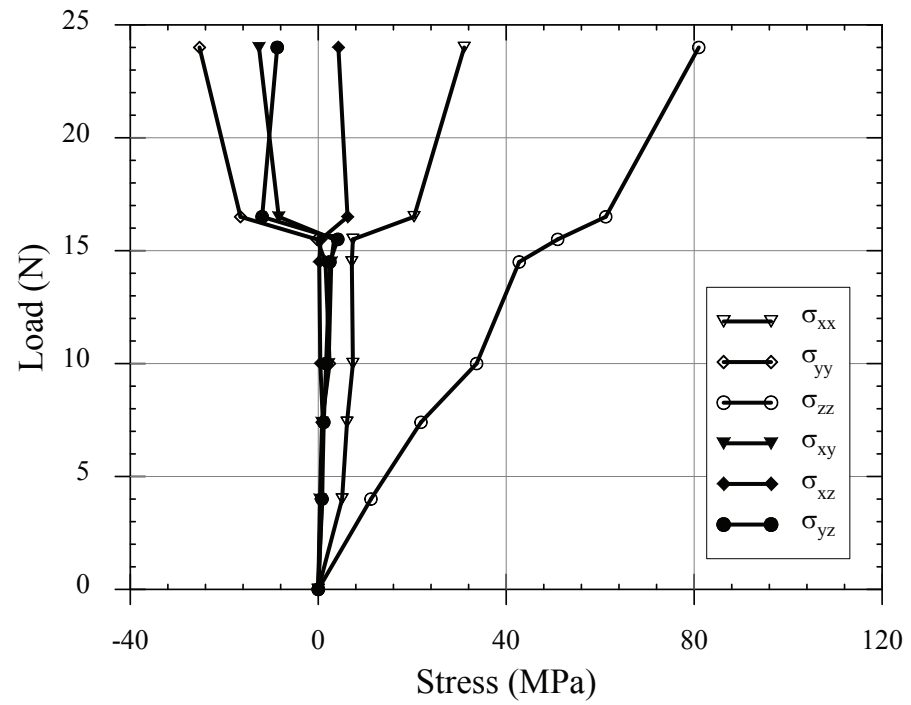

(a)

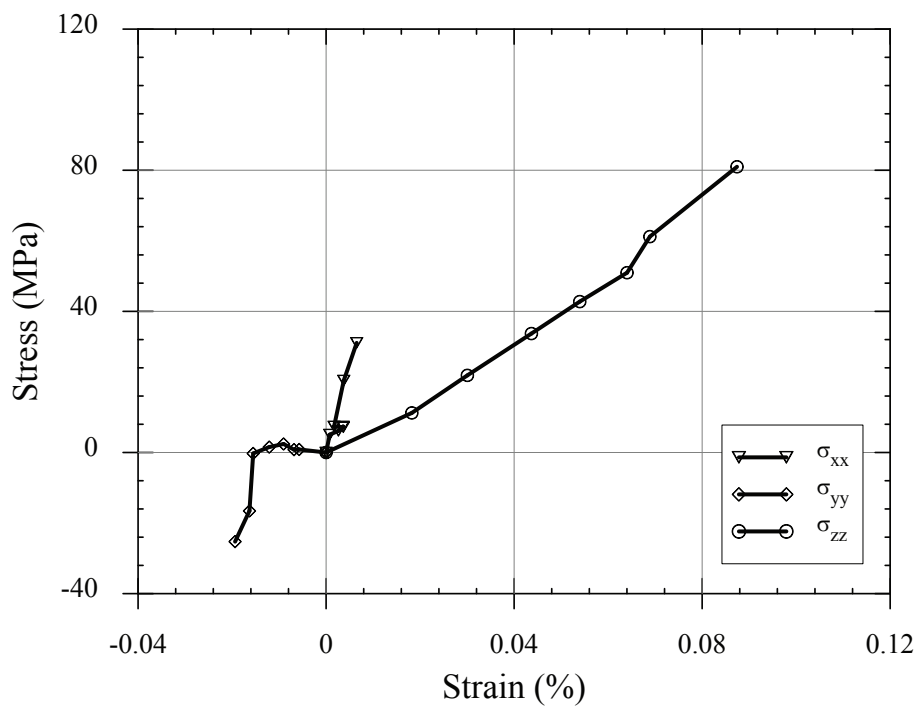

(b)

Figure 11. (a) Applied load versus sample frame stress tensor components of the middle particle of Test 3, and (b) stress versus strain variation for the middle particle of Test 3. 